\title{
Numerical modelling of backward front propagation in piping erosion by DEM-LBM coupling
}

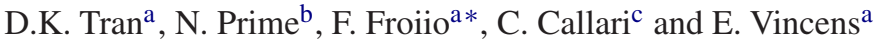 \\ ${ }^{a}$ LTDS, Univ Lyon, École Centrale de Lyon, Ecully, France; ${ }^{b}$ LOCIE, Université Savoie Mont Blanc, \\ Le Bourget du Lac, France; ${ }^{c}$ DiBT, Università del Molise, Campobasso, Italy
}

(Received 23 May 2016; accepted 7 October 2016)

\begin{abstract}
A granular soil REV located on the upstream side of the erosion pipe front is modelled numerically, at the grain scale, by coupling the Discrete Element Method (DEM) with the Lattice Boltzmann Method (LBM) for the representation of the solid and fluid phases, respectively. The implementation of DEM follows a standard molecular dynamics approach and the interactions between grains are regulated by unilateral contacts and breakable bonds. A synopsis of the LBM scheme is provided, with focus on the implementation of non-slip conditions for moving boundaries and use of the Multiple Relaxation Time approach for improved numerical stability. The coupling scheme is described along with the criteria for setting the numerical parameters of the two methods. After a "dry" preparation procedure, the numerical REV is tested under fully saturated conditions and increasing pressure difference. Backward erosion is observed and a micromechanical inspection of the granular phase suggests that arching through force chains and the breakage of tensile bonds are prominent resistance and degradation mechanisms, respectively.
\end{abstract}

Keywords: backward erosion; piping erosion; discrete element method; lattice Boltzmann method

\section{Introduction}

Piping erosion in the foundations of hydraulic works or within embankment dams and dykes is a frequent and highly damaging phenomenon (da Silveira, 1984; Yen \& Tung, 1993; Zhang, Xu, \& Jia, 2009), which motivates a major concern for basin authorities as well as for companies exploiting water resources. A convenient description of the phenomenon rests upon the distinction of four ordered phases of evolution: initiation, continuation, progression and breaching (Fell, Foster, \& Wan, 2007; Fell \& Fry, 2007). Piping erosion may be initiated within the embankment or its foundation due to concentrated leaks, suffusion, backward erosion (e.g. at the downstream face of the embankment or of the embankment core) or due to other hydro-erosion mechanisms. The continuation phase depends on the presence of filters able to prevent the triggering mechanism from evolving at a larger scale and forming a pipe. In the progression phase, a formed pipe propagates towards the reservoir, while enlarging, until the process results into a breach, i.e. in the uncontrolled loss of storage, possibly in connection with instability phenomena (e.g. collapse of the pipe, slope instability or overtopping due to settlement of the embankment).

In the last few decades, the increase in hazard and vulnerability due to climate change as well as the call for a more sustainable exploitation of water resources motivated a larger effort

\footnotetext{
*Corresponding author. Email: francesco.froiio@ec-lyon.fr
} 
on the experimental observation and on the modelling of hydro-erosion mechanisms, with particular attention for those involved in piping erosion (see Bonelli, 2013; Cheng, Draper, $\&$ An, 2015 and references therein). In this process, two different erosion mechanisms can be singled out in a rough picture: the enlargement of the pipe is regulated by tangential erosion caused at the pipe walls by the turbulent flow, while backward erosion induced by Darcy flow prevails at the upstream-propagating pipe tip. Several experimental, analytical and numerical studies on the tangential erosion mechanism have lead to substantial advances in the understanding and modelling of the pipe enlargement kinetics (Bonelli \& Brivois, 2008; Cottereau, Díez, \& Huerta, 2010; Lominé, Scholtès, Sibille, \& Poullain, 2013; Wan \& Fell, 2002, 2004). On the other hand, few experimental works concern the backward erosion mechanism (Sellmeijer, de la Cruz, van Beek, \& Knoeff, 2011; van Beek, Knoeff, \& Sellmeijer, 2011), on which there is a remarkable absence of analytical and numerical studies. The gap is partially filled by previous modelling of the sand production problem (Papamichos \& Vardoulakis, 2005; Vardoulakis, Papanastasiou, \& Stavropoulou, 2001; Vardoulakis, Stavropoulou, \& Papanastasiou, 1996), as a closely related erosion process induced by interstitial laminar flow. However, models specifically proposed for localised backward erosion in porous media are still very rare (Rotunno, Callari, \& Froiio, in preparation; Wang, Fu, Jie, Dong, \& Hu, 2014).

This study aims at calling attention on the conceptual and numerical tools of micromechanics of granular materials (Cambou, Jean, \& Radjai, 2009) for their application to the understanding and modelling of piping erosion. Among those tools, the Discrete Element Method (DEM) is nowadays a standard numerical approach (Radjai \& Dubois, 2011) and its coupling with several candidate methods from computational fluid dynamics is being proposed for the modelling of fluid-saturated granular materials.

According to a first class of coupled approaches, two separate scales are juxtaposed for the solid and the fluid phases, i.e. the discrete scale of the single grain and the continuum scale of Darcy flow, respectively (McNamara, Flekkøy, \& Måløy, 2002; Zeghal \& El Shamy, 2002). Other coupling schemes rest upon the discretisation of the fluid flow at the scale of the pores (Chareyre, Cortis, Catalano, \& Barthélemy, 2011), or at scales smaller than the representative grain size (Ladd, 1994a, 1994b; Wachs, 2009). Among the latter, the coupling between DEM and the lattice Boltzmann method (LBM) is receiving increasing attention and provides space-time resolution of the fluid flow required for modelling hydro-erosion phenomena, i.e. of physical situations in which the fluid domain is characterised by complex geometries and may evolve abruptly (Cui, Li, Chan, \& Chapman, 2012; Mansouri, Delenne, El Youssoufi, \& Seridi, 2009).

With regard to piping erosion, coupling with LBM has recently been employed for DEM modelling of tangential erosion at the walls of the conduit (Lominé et al., 2013; Sibille, Lominé, Poullain, Sail, \& Marot, 2015). Actually, the same numerical tools are employed in the numerical simulation presented in this study, designed for the micromechanical investigation of backward erosion at the pipe front.

Section 2 reports on the DEM and LBM schemes, and their coupling, as implemented in the in-house 2D code used in this study. The numerical simulation procedure is described in Section 3, along with a micromechanical inspection of the results, before the paper is closed with some summary remarks. 


\section{Discrete numerical modelling}

\subsection{Granular skeleton}

\subsubsection{Discrete element approach}

DEM has emerged as an effective numerical approach for modelling the mechanical behaviour of granular media such as soils, particularly as a tool for numerical experiments at scales close to the REV and in complement to laboratory tests. An in-house 2D DEM code was used in this study to investigate the micromechanical (grain-scale) processes underwent by the granular skeleton in the pipe tip region.

The code was developed following a standard molecular dynamics approach (Cundall $\&$ Strack, 1979). Grains are represented by circular discs and their interactions are modelled by enabling normal and tangential forces at the contact points. Such forces (referred to as $\mathbf{F}_{\mathrm{n}}$ and $\mathbf{F}_{\mathrm{t}}$, resp.) can result from either a bond between the two grains or a unilateral contact. The resultant force system on each grain, relative to its mass center, is determined based on the interaction with neighbouring grains, computed as above, and with the surrounding fluid, as detailed in Section 2.2. Gravity is neglected. The trajectory of each grain, including rotation, is computed by time integration of the laws of motion for rigid bodies, via the standard Störmer-Verlet discretisation scheme (Cundall \& Strack, 1979).

\subsubsection{Grain-to-grain interactions}

Prior to the failure of a bond, the interaction between the two grains is bilateral and viscoelastic, i.e. according to Figure 1,

$$
\begin{aligned}
& \mathbf{F}_{\mathrm{n}}=F_{\mathrm{n}} \mathbf{n}, \quad \text { with } \quad F_{\mathrm{n}}=-k_{\mathrm{n}} \Delta x_{\mathrm{n}}-c_{\mathrm{n}}\left(\Delta \mathbf{v}_{\mathrm{c}} \cdot \mathbf{n}\right) \\
& \mathbf{F}_{\mathrm{t}}=F_{\mathrm{t}} \mathbf{t}, \quad \text { with } \quad F_{\mathrm{t}}=-k_{\mathrm{t}} \Delta x_{\mathrm{t}}-c_{\mathrm{t}}\left(\Delta \mathbf{v}_{\mathrm{c}} \cdot \mathbf{t}\right)
\end{aligned}
$$

where $\Delta \mathbf{v}_{\mathrm{c}}=\mathbf{v}_{\mathrm{c}}^{(1)}-\mathbf{v}_{\mathrm{c}}^{(2)}$ is the relative velocity of the two representative contact points; $\mathbf{n}$ is the normal unit vector at the contact point, directed as the segment joining the centers of the two grains and oriented from grain 1 to grain 2 in the figure; $\mathbf{t}$ is the tangential unit vector obtained from $\mathbf{n}$ after a counterclockwise rotation; $\Delta x_{\mathrm{n}}$ and $\Delta x_{\mathrm{t}}$, obtained by time integration of $\Delta \mathbf{v}_{\mathrm{c}} \cdot \mathbf{n}$ and $\Delta \mathbf{v}_{\mathrm{c}} \cdot \mathbf{t}$, are the normal and tangential cumulative relative displacements, respectively; $k_{\mathrm{n}}$ and $k_{\mathrm{t}}$ are elastic stiffnesses while $c_{\mathrm{n}}$ and $c_{\mathrm{t}}$ are viscous constants in the normal and tangential directions, respectively. The corresponding rheological model is sketched in Figure 2(a). The failure envelope in Figure 2(b) (continuous line) characterises the tensile and shear strengths of the bond: the latter is maintained as long as

$$
F_{\mathrm{n}}>-A \text { and }\left|F_{\mathrm{t}}\right|<\mu F_{\mathrm{n}}+C
$$

where the strength parameters $A>0$ (bond adhesion), $C>0$ (bond cohesion) and $\mu>0$ (contact friction) are chosen such that $C \geq \mu A$.

As the bond breaks, the normal interaction becomes unilateral and the tangential force limit is computed on a purely frictional basis (see Figure 2(c) and the dashed line in Figure 2(b)):

$$
\begin{aligned}
& F_{\mathrm{n}}=-k_{\mathrm{n}} \Delta x_{\mathrm{n}}-c_{\mathrm{n}}\left(\Delta \mathbf{v}_{\mathrm{c}} \cdot \mathbf{n}\right) \\
& F_{\mathrm{n}}=0
\end{aligned}
$$$$
\text { for }
$$$$
\Delta x_{\mathrm{n}} \geq 0
$$

for

and

$$
F_{\mathrm{t}}=\overbrace{-k_{\mathrm{t}} \Delta x_{\mathrm{t}}-c_{\mathrm{t}}\left(\Delta \mathbf{v}_{\mathrm{c}} \cdot \mathbf{t}\right)}^{=: F_{\mathrm{t}}^{*}}, \quad \text { if }\left|F_{\mathrm{t}}^{*}\right| \leq \mu F_{\mathrm{n}}
$$




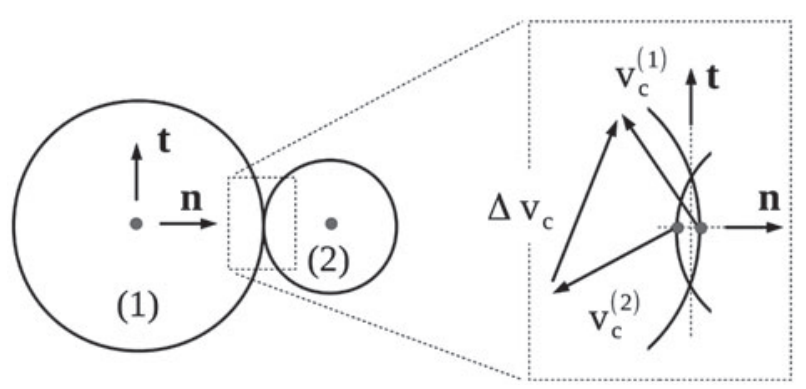

Figure 1. Normal and tangential unit vectors ( $\mathbf{n}$ and $\mathbf{t}$, resp.) at the oriented contact between grain 1 and 2. Relative velocity $\Delta \mathbf{v}_{\mathrm{c}}$ at the oriented contact vs. the velocities $\mathbf{v}_{\mathrm{c}}^{(1)}$ and $\mathbf{v}_{\mathrm{c}}^{(2)}$ of the representative contact points of grain 1 and grain 2 , respectively.

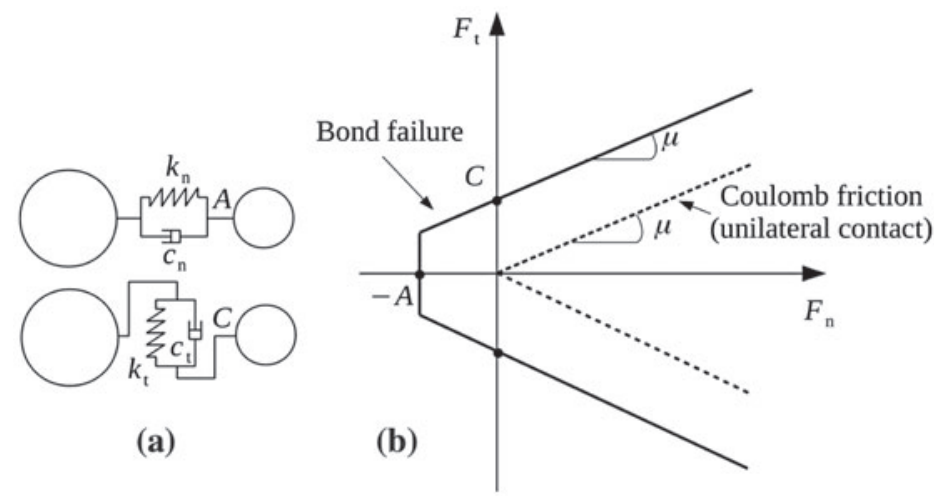

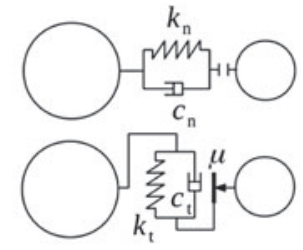

(c)

Figure 2. Rheological models: for grain-to-grain bonds in (a) and (b), continuous line; for unilateral grain-to-grain contacts in (c) and (b), dashed line.

$$
F_{\mathrm{t}}=\operatorname{sign}\left(-k_{\mathrm{t}} \Delta x_{\mathrm{t}}-c_{\mathrm{t}}\left(\Delta \mathbf{v}_{\mathrm{c}} \cdot \mathbf{t}\right)\right)\left|F_{\mathrm{n}} \mu\right|, \quad \text { if }\left|F_{\mathrm{t}}^{*}\right|>\mu F_{\mathrm{n}}
$$

A more refined model might of course distinguish between the role played by $\mu$ in (3) and in (6), i.e. as a strength parameter and as a contact friction parameter, respectively.

\subsubsection{Rigid-wall boundary conditions}

Rigid walls, as used in this study for the confinement of the granular assembly, are modelled as straight lines endowed with translational and rotational degrees of freedom (the rigid motion being tracked through a reference point moving along with the wall). The interaction between a wall and the grains, referred to herein as wall-to-grain interaction, is described by the same model as for grain-to-grain interactions, provided the relative contact velocity $\Delta \mathbf{v}_{\mathrm{c}}$ is defined according to the necessary adaptation of Figure 1. 


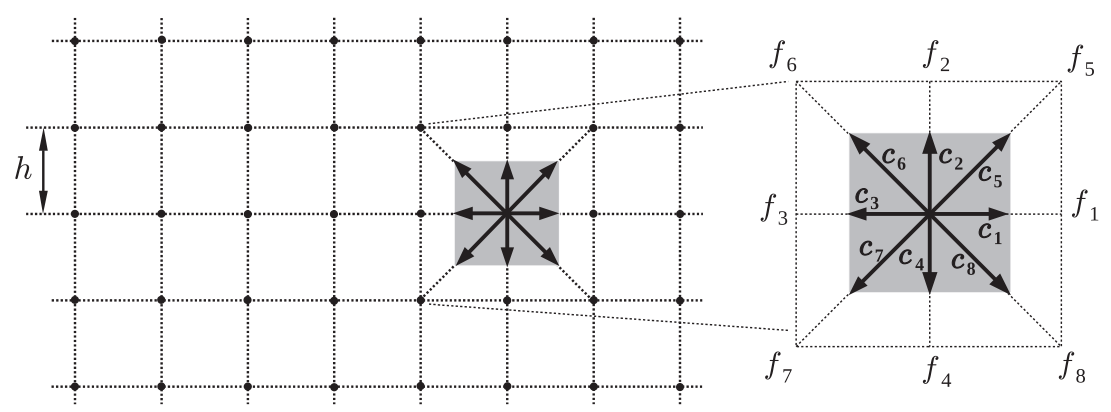

Figure 3. D2Q9 lattice model.

\subsection{Pore fluid}

\subsubsection{Standard LBM algorithm}

Introduced in 1988 by McNamara and Zanetti (1988), LBM was first developed as a statistical upscaling of lattice-gas cellular automota (LGCA) in order to overcome several drawbacks of this previous method as a candidate technique for fluid dynamics computations (e.g. statistical noise Frisch et al., 1987; Frisch, Hasslacher, \& Pomeau, 1986; Wolfram, 1994). However, LBM can be derived directly from Boltzmann equation (He \& Luo, 1997a) and was shown to be equivalent to a finite difference approximation to Navier-Stokes equations for incompressible fluids (Frisch, 1989). In the remainder of this section, the key elements of the method are recalled, including the core algorithm and some techniques for the implementation of the boundary conditions (no-slip boundaries and constant pressure boundaries) employed in this work. The synthetic description also includes a presentation of the applied scheme for the coupling with DEM.

LBM is a kinetic theory-based numerical approach to fluid dynamics problems. The method is hinged upon a discrete form of Boltzmann equation and therefore on the determination of the distribution function $f(\mathbf{x}, \mathbf{c}, t)$, defined as the probability density for the presence of a fluid particle with velocity $\mathbf{c}$ at position $\mathbf{x}$, as random variables and time $t$, as a parameter. To solve $f$ numerically, time is segmented in time intervals of fixed amplitude $\Delta t$ and the fluid domain is discretised onto a regular lattice. The case of the two-dimensional rectangular lattice D2Q9 (Qian, D'Humières, \& Lallemand, 1992) used in this study is illustrated in Figure 3, where $h$ is the spacing between neighbouring lattice nodes. As shown in the same figure, the velocity space is also discretised accordingly, i.e. reduced to the set $\left\{\mathbf{c}_{\alpha}\right\}_{\alpha=0,1, \ldots, N}$ of velocity vectors enabling the fluid particles to hop from one lattice node on any of its $N=8$ neighbouring nodes (or to stay at the current position) in a time interval $\Delta t$. The relevant velocity vectors for the D2Q9 model are

$$
\mathbf{c}_{\alpha}= \begin{cases}0 & \alpha=0 \\ c\left(\cos \frac{(\alpha-1) \pi}{2} \mathbf{e}_{1}+\sin \frac{(\alpha-1) \pi}{2} \mathbf{e}_{2}\right), & \alpha=1,2,3,4 \\ \sqrt{2} c\left(\cos \frac{(2 \alpha-9) \pi}{4} \mathbf{e}_{1}+\sin \frac{(2 \alpha-9) \pi}{4} \mathbf{e}_{2}\right), & \alpha=5,6,7,8\end{cases}
$$

where $\mathbf{e}_{1}$ and $\mathbf{e}_{2}$ are the unit vectors of a Cartesian reference system oriented according to the main lattice directions (i.e. the horizontal and vertical directions in the Figure 3), while $c=h / \Delta t$ is the characteristic lattice speed. 
Consistent with the above discretisation, at each lattice node the distribution function takes only $N+1$ values $f_{\alpha}$, with the index $\alpha$ spanning the velocity values. In this setting, one can refer to

$$
f_{\alpha}\left(\mathbf{x}+\mathbf{c}_{\alpha} \Delta t, t+\Delta t\right)-f_{\alpha}(\mathbf{x}, t)=-\frac{\Delta t}{\tau}\left(f_{\alpha}(\mathbf{x}, t)-f_{\alpha}^{e q}(\mathbf{x}, t)\right)
$$

as the discrete counterpart of a simplified form of Boltzmann equation:

$$
\frac{\partial f(\mathbf{x}, \mathbf{c}, t)}{\partial t}+\frac{\partial f(\mathbf{x}, \mathbf{c}, t)}{\partial \mathbf{x}} \cdot \mathbf{c}=-\frac{f(\mathbf{x}, \mathbf{c}, t)-f^{\mathrm{eq}}(\mathbf{x}, \mathbf{c}, t)}{\tau}
$$

in which $\mathbf{c}$ is the microscopic fluid particle velocity, $f^{\mathrm{eq}}$ is the distribution function at thermodynamic equilibrium (the Maxwell distribution is used) while the parameter $\tau$, called relaxation time, is a measure of the promptness by which the fluid relaxes towards thermodynamic equilibrium after a perturbation of the latter. The terms on the r.h.s. of (9) and (10) are the relevant statistical models for the collisions between fluid particles; namely, (10) is obtained from the original Boltzmann equation, following the BhatnagarGross-Krook approximation to the original collision operator, based on the assumption of small perturbations in thermodynamic equilibrium (Bhatnagar, Gross, \& Krook, 1954). Notice, finally, that negligible body forces are assumed in (9) and (10).

Basic macroscopic quantities such as the mass density $\rho$ are retrieved at each node as customary in statistical mechanics, by computation of velocity moments, of the appropriate order, of the distribution function. Denoting by $m$ the mass of the fluid particle, mass and momentum densities can be computed as:

$$
\rho=m \sum_{\alpha=0}^{8} f_{\alpha}, \quad \rho \mathbf{u}=m \sum_{\alpha=0}^{8} f_{\alpha} \mathbf{c}_{\alpha}
$$

respectively; or the stress tensor $\sigma$ can be computed as:

$$
\boldsymbol{\sigma}=m \sum_{\alpha=0}^{8} f_{\alpha} \mathbf{c}_{\alpha} \otimes \mathbf{c}_{\alpha}
$$

With the aim to simulate nearly incompressible flows, the method can be specialised to the low Mach number regime, i.e. for Ma $\ll 1$, where Ma $=\|\mathbf{u}\| / c$ is the so-called computational Mach number (the sound speed in the lattice being $c_{\mathrm{S}}=c / \sqrt{3}$ ).

In the limit as $\mathrm{Ma} \rightarrow 0$, the Maxwell distribution function can be expressed as:

$$
f_{\alpha}^{e q}=w_{\alpha} \frac{\rho}{m}\left(1+3 \frac{\mathbf{c}_{\alpha} \cdot \mathbf{u}}{c^{2}}+\frac{9}{2} \frac{\left(\mathbf{c}_{\alpha} \cdot \mathbf{u}\right)^{2}}{c^{4}}-\frac{3}{2} \frac{\|\mathbf{u}\|^{2}}{c^{2}}\right)+\mathcal{O}\left(\mathrm{Ma}^{3}\right)
$$

with

$$
w_{\alpha}=\left\{\begin{array}{l}
\frac{4}{9} \quad \alpha=0 \\
\frac{1}{9} \quad \alpha=1,2,3,4 \\
\frac{1}{36} \alpha=5,6,7,8
\end{array}\right.
$$

Under the assumption of small variations in mass density, i.e.

$$
\frac{\rho-\rho_{0}}{\rho_{0}}=\mathcal{O}\left(\mathrm{Ma}^{2}\right)
$$

where $\rho_{0}$ is a reference value, a derivation based on the Chapman-Enskog asymptotic expansion procedure (He \& Luo, 1997b) identifies the corresponding model in the continuum 
limit as the Navier-Stokes equations for incompressible fluids, under negligible body forces, up to an error of the order $\mathcal{O}\left(\mathrm{Ma}^{2}\right)$ on the mass balance and $\mathcal{O}\left(\mathrm{Ma}^{3}\right)$ on the momentum balance:

$$
\begin{gathered}
\operatorname{div} \mathbf{u}=\mathcal{O}\left(\mathrm{Ma}^{2}\right) \\
\rho_{0}\left(\frac{\partial \mathbf{u}}{\partial t}+(\operatorname{grad} \mathbf{u}) \mathbf{u}\right)=-\operatorname{grad} p+\eta \operatorname{div} \operatorname{grad} \mathbf{u}+\mathcal{O}\left(\mathrm{Ma}^{3}\right)
\end{gathered}
$$

where

$$
p=c_{\mathrm{s}}^{2} \rho, \quad \eta=\frac{\rho_{0}}{3}\left(\frac{\tau}{\Delta t}-\frac{1}{2}\right) \frac{h^{2}}{\Delta t}
$$

are the consistent definitions for the pressure and the dynamic viscosity, respectively. ${ }^{1}$

\subsubsection{Collision and streaming time-substeps}

It is of practical convenience to reorganise and decompose (9) as:

$$
\begin{aligned}
f_{\alpha}^{\mathrm{c}}(\mathbf{x}, t) & =f_{\alpha}(\mathbf{x}, t)-\frac{\Delta t}{\tau}\left(f_{\alpha}(\mathbf{x}, t)-f_{\alpha}^{\mathrm{eq}}(\mathbf{x}, t)\right) \\
f_{\alpha}\left(\mathbf{x}+\mathbf{c}_{\alpha} \Delta t, t+\Delta t\right) & =f_{\alpha}^{\mathrm{c}}(\mathbf{x}, t)
\end{aligned}
$$

where the post-collision discrete distribution function $f_{\alpha}^{\mathrm{c}}(\mathbf{x}, t)$, defined in (16), carries the values of $f_{\alpha}(\mathbf{x}, t)$ as affected at the relevant node by the collisions taking place "simultaneously" and instantaneously at $t$. The advection of fluid particles, during $\Delta t$, is then taken into account separately by (17). The above decomposition is almost dictated by the numerical implementation of the method, due to the simplification of the algorithm allowed by the definition of separated collision and streaming time substeps, in reference to (16) and (17), respectively.

\subsubsection{Multiple relaxation time approach}

According to (9) and (10), the relaxation of the distribution function is controlled by a single parameter (i.e. the relaxation time $\tau$ ). Some drawbacks of this attractive but strong simplification, among which a certain degree of numerical instability, were resolved by its generalisation towards Multiple Relaxation Times (MRT) (d'Humière, 1992; d'Humière, Ginzburg, Krafczyk, Lallemand, \& Luo, 2002; Lallemand \& Luo, 2000). The latter approach is based on the assumption that different velocity moments of the distribution function relax linearly at different time rates. The relevant moments are assembled as polynomials of previously defined quantities (mass and momentum densities, stress) and of additional energy-related terms; they provide the same information as carried by the values of the distribution function, i.e. 


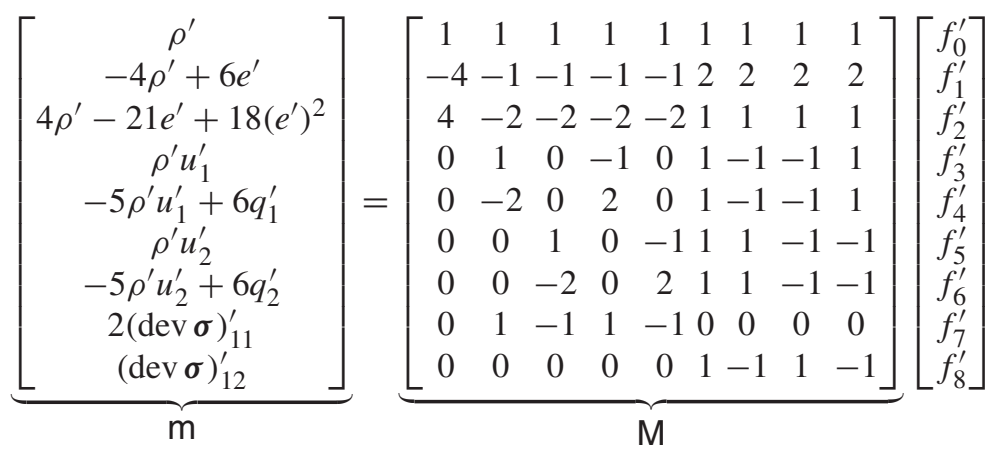

where the transformation matrix $\mathrm{M}$ is invertible and in which the prime symbol denotes dimensionless quantities obtained, from the relevant metric quantity, after normalisation by the lattice units (i.e. $m, h$ and $\Delta t$ ). In (18), $e$ is the thermal energy density, $q_{1}$ and $q_{2}$ are Cartesian components of the heat flux vector and $\operatorname{dev} \sigma$ is the deviatoric (viscous) part of the stress tensor $\sigma .^{2}$

According to (18), the single-relaxation-time Equation (9) is replaced by

$$
f_{\alpha}^{\prime}\left(\mathbf{x}+\mathbf{c}_{\alpha} \Delta t, t+\Delta t\right)-f_{\alpha}^{\prime}(\mathbf{x}, t)=-\sum_{\beta, \gamma=0}^{8} M_{\alpha \beta}^{-1} S_{\beta \gamma}\left(m_{\gamma}(\mathbf{x}, t)-m_{\gamma}^{e q}(\mathbf{x}, t)\right)
$$

where $M_{\alpha \beta}^{-1}$ and $m_{\gamma}$ denote the coefficients of $\mathrm{M}^{-1}$ and the elements of $\mathrm{m}$, respectively; the scalars $m_{\gamma}^{e q}$ are computed as the $m_{\gamma}$ but based on the Maxwell distribution function. In the same equation, $S_{\beta \gamma}$ are the coefficients of the so-called diagonal relaxation matrix S, defined as

$$
\mathrm{S}=\operatorname{diag}\left(s_{0}, s_{1}, s_{2}, s_{3}, s_{4}, s_{5}, s_{6}, s_{7}, s_{8}\right)
$$

which can be seen as a generalisation of the relaxation parameter $\Delta t / \tau$ in (9). Consistently, (19) reduces to (9) if $s_{0}=s_{1}=\cdots=s_{8}=\Delta t / \tau$. It is worth mentioning anyway that $s_{0}$, $s_{3}$ and $s_{5}$ have no effect on the computation, as these eigenvalues are directly related to the collision invariants $\rho$ and $\rho \mathbf{u}$. The remaining parameters are chosen in the interval $(0,2)$ for numerical stability.

Finally, the Chapman-Enskog asymptotic expansion of the MRT model delivers once more the Navier-Stokes equations for incompressible fluids (Du, Shi, \& Chen, 2006), i.e. (9)-(15), provided one sets $s_{7}=s_{8}$ (to enforce isotropy) and the dynamic viscosity is identified as

$$
\eta=\frac{\rho_{0}}{3}\left(\frac{1}{s_{7}}-\frac{1}{2}\right) \frac{h^{2}}{\Delta t}=\frac{\rho_{0}}{3}\left(\frac{1}{s_{8}}-\frac{1}{2}\right) \frac{h^{2}}{\Delta t}
$$

\subsubsection{No-slip condition for moving boundaries}

No-slip conditions at stationary as well as moving solid boundaries, straight or curved, can be imposed through the standard "bounce-back" scheme (Aidun, Lu, \& Ding, 1998; Ladd, 1994a); the actual position of the boundary is discretised on the lattice grid at each time step of the LBM algorithm and the effect of its motion is computed in terms of momentum transfer between the fluid and the solid phases. This method, though effective in ensuring no tangential velocity along the fluid-solid boundary, results in geometric discontinuities, accompanying the motion of the interface, that produce important fluctuations in the computed hydrodynamic forces. A regularised model was proposed in Bouzidi, Firdaouss, and Lallemand (2001) and is based on an interpolation procedure enabling to track the boundary 
at arbitrary sub-grid positions, with improved numerical stability. ${ }^{3}$ To introduce this method, used in the present study, Figure 4 illustrates the case of a fluid-solid boundary identified as the circular profile of a grain. The white and black circular marks in the figure are located at grid nodes in the fluid and solid regions, respectively. The gray circular marks denote the position (e.g. $\mathbf{x}_{\mathrm{b}}$ ) at which the boundary is crossed by the path from a fluid node to a neighbouring solid node ( $\mathbf{x}_{\mathrm{f}}$ and $\mathbf{x}_{\mathrm{s}}$, resp.). The fluid particles accounted for in the collisions step at $\mathbf{x}_{\mathrm{f}}$ are advected from all the neighbouring nodes but those in the solid region. The missing contributions are computed based on the following heuristic argument, illustrated for $\mathbf{x}_{\mathrm{f}}$ and $\mathbf{x}_{\mathrm{s}}$ in the figure: a fluid particle that is delivered at $\mathbf{x}_{\mathrm{f}}$ after bouncing on the boundary at $\mathbf{x}_{\mathrm{b}}$ would be located, one time step before, at the fictitious fluid node $\mathbf{x}_{\mathrm{ff}}$, i.e. at a distance compatible with the path of the particle and the velocities allowed by the lattice model. Let

as in the figure, then the fluid node is located at

$$
q=\frac{\left\|\mathbf{x}_{\mathrm{f}}-\mathbf{x}_{\mathrm{b}}\right\|}{\left\|\mathbf{x}_{\mathrm{f}}-\mathbf{x}_{\mathrm{s}}\right\|}<\frac{1}{2}
$$

$$
\mathbf{x}_{\mathrm{ff}}=2 q \mathbf{x}_{\mathrm{f}}+(1-2 q)\left(\mathbf{x}_{\mathrm{f}}-\Delta t \mathbf{c}_{8}\right)
$$

where $\mathbf{x}_{\mathrm{f}}-\Delta t \mathbf{c}_{8}$ is the position of the fluid node back off $\mathbf{x}_{\mathrm{f}}$. Consistently, the relevant missing contribution to the distribution function at $\mathbf{x}_{\mathrm{f}}$ is computed (at time $t+\Delta t$ ) as

$$
f_{6}\left(\mathbf{x}_{\mathrm{f}}, t+\Delta t\right)=\underbrace{2 q f_{8}^{\mathrm{c}}\left(\mathbf{x}_{\mathrm{f}}, t\right)+(1-2 q) f_{8}^{\mathrm{c}}\left(\mathbf{x}_{\mathrm{f}}-\Delta t \mathbf{c}_{8}, t\right)}_{f_{8}^{\mathrm{c}}\left(\mathbf{x}_{\mathrm{ff}}, t\right)}+\frac{6 \omega_{8} \rho}{m c^{2}} \mathbf{c}_{8} \cdot \mathbf{v}_{\mathrm{b}}
$$

where the post-collision value $f_{8}^{\mathrm{c}}\left(\mathbf{x}_{\mathrm{ff}}, t\right)$ of the distribution function at the fictitious fluid node is computed by interpolation on the neighbouring nodes, and the additional (last) term on the r.h.s. is representative of the momentum transfer due to the velocity $\mathbf{v}_{\mathrm{b}}$ of the boundary at $\mathbf{x}_{\mathrm{b}}$. The generalisation to arbitrary relative positions of the fluid-, solid- and boundary nodes is

$$
f_{\alpha}\left(\mathbf{x}_{\mathrm{f}}, t+\Delta t\right)= \begin{cases}2 q f_{\hat{\alpha}}^{\mathrm{c}}\left(\mathbf{x}_{\mathrm{f}}, t\right)+(1-2 q) f_{\hat{\alpha}}^{\mathrm{c}}\left(\mathbf{x}_{\mathrm{f}}-\Delta t \mathbf{c}_{\hat{\alpha}}, t\right)+\frac{6 \omega_{\hat{\alpha}} \rho}{m c^{2}} \mathbf{c}_{\hat{\alpha}} \cdot \mathbf{v}_{\mathrm{b}}, q<\frac{1}{2} \\ \frac{1}{2 q} f_{\hat{\alpha}}^{\mathrm{c}}\left(\mathbf{x}_{\mathrm{f}}, t\right)+\frac{2 q-1}{2 q} f_{\alpha}^{\mathrm{c}}\left(\mathbf{x}_{\mathrm{f}}, t\right)+\frac{3 \omega_{\hat{\alpha}} \rho}{q m c^{2}} \mathbf{c}_{\hat{\alpha}} \cdot \mathbf{v}_{\mathrm{b}}, & q \geq \frac{1}{2}\end{cases}
$$

in which the expression for $q \geq 1 / 2$ stems for a similar reasoning and the index $\hat{\alpha}$ is such that $\mathbf{c}_{\hat{\alpha}}=-\mathbf{c}_{\alpha}$.

An issue related to the implementation of moving impervious boundaries is the conversion of solid nodes into fluid nodes due to the displacement of the boundary between two time steps. Namely, the issue concerns the values of the distribution function at the "uncovered" fluid nodes. As proposed in Aidun et al. (1998), the latter is initialised to the values of the equilibrium distribution function $f_{\alpha}^{\text {eq }}$, i.e. the mass density $\rho$ in (12) is computed by interpolation on the neighbouring fluid nodes, while the macroscopic fluid velocity $\mathbf{u}$ in the same expression is obtained by extension, to the fluid node, of the rigid motion of the boundary. ${ }^{4}$ Conversely, the fluid nodes that are turned into solid nodes are temporarily excluded from the core LBM algorithm described in Sections 2.2.1-2.2.3. However, with the conservation of fluid mass not being an implicit feature of this scheme, the relevant accuracy is to be checked throughout the computation (see Section 3.2).

\subsubsection{Pressure boundary condition}

This condition is assigned at the two opposite, inlet and outlet sections of the rectangular flow channel (otherwise confined by no-slip boundaries) designed for the numerical simulation 


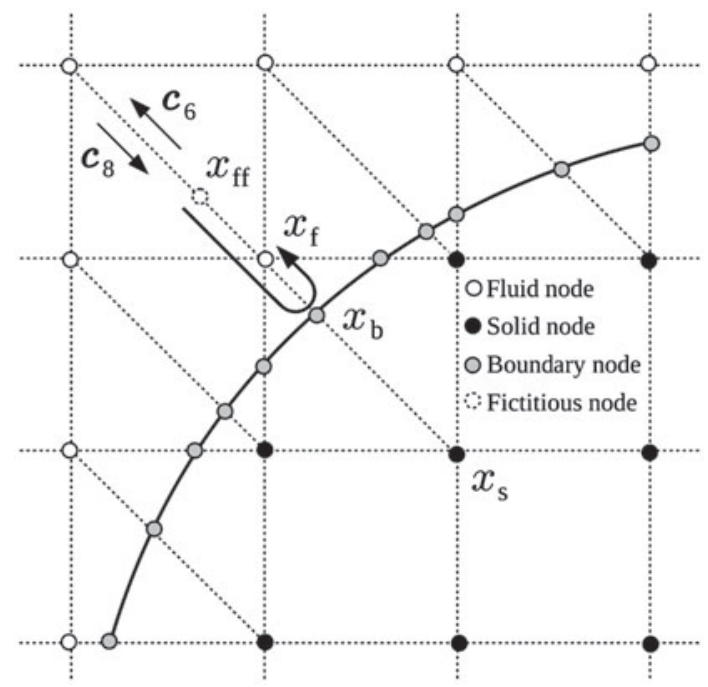

Figure 4. Lattice grid at a curved fluid-solid boundary.

described in Section 3. In LBM simulations, the pressure $p$ as well as the velocity $\mathbf{u}$ of the flow cannot be assigned directly, but through the values of the discrete distribution function at the relevant boundary node. The case of an inlet node is sketched in Figure 5: $f_{1}, f_{5}$ and $f_{8}$ are undetermined and can be adjusted in order to enforce the inlet pressure $p_{\text {in }}$, while the remaining values of the discrete distribution function are determined after the streaming step as a result of advection from inner nodes. As a first assignment on $f_{1}, f_{5}$ and $f_{8}$, the inlet pressure $p_{\text {in }}$ can be imposed directly through the condition

$$
m \sum_{\alpha=0}^{8} f_{\alpha}=\rho=\frac{p_{\text {in }}}{c_{\mathrm{s}}^{2}}
$$

according to (11) and (15). A second condition can be deduced from the assumption of null component of the velocity parallel to the boundary and can be specified as

$$
\sum_{\alpha=0}^{8} f_{\alpha} \mathbf{c}_{\alpha} \cdot \mathbf{e}_{2}=0
$$

consistently with (11) and with the orientation of the inlet boundary in the figure. The third condition required to close the system is usually set as proposed in Zou and He (1997), i.e.

$$
f_{1}-f_{1}^{\mathrm{eq}}=f_{3}-f_{3}^{\mathrm{eq}}
$$

which can be read as a bounce-back rule on the non equilibrium part of the discrete distribution function. The same method applies for the generic outlet node and can be adapted for nodes at the intersection between pressure and no-slip boundaries.

\subsubsection{Validation example: flow past a fixed circular disc}

A validation of the LBM algorithm is proposed, based on the boundary value problem in Figure 6(a). A parabolic profile of horizontal macroscopic fluid velocity is assigned at the 


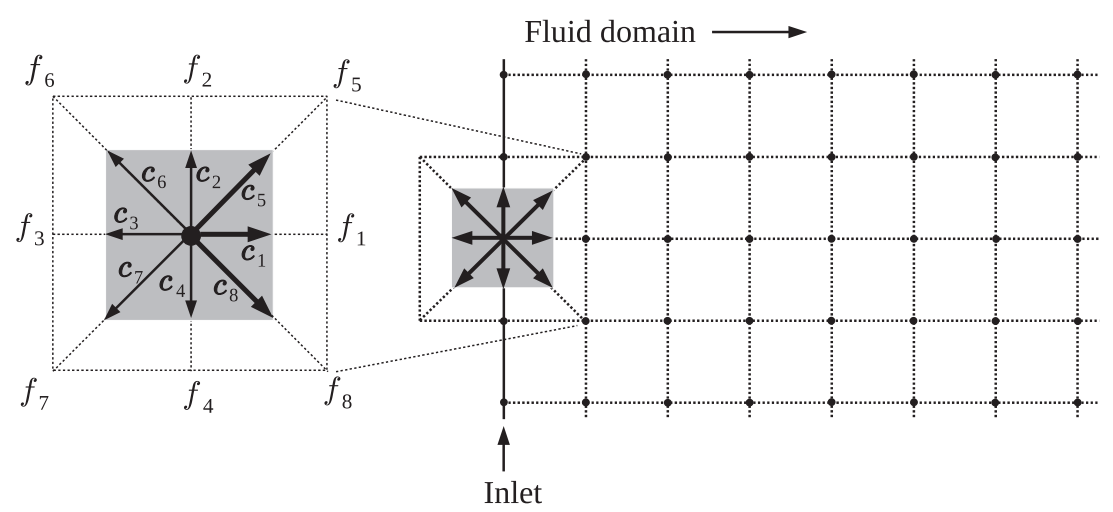

Figure 5. Generic node at the inlet boundary.

Table 1. Parameters employed for the numerical solution of the boundary value problem in Figure 6(a). The eigenvalues $s_{0}, s_{3}$ and $s_{5}$ of the relaxation matrix are not reported as they have no effect on the simulation (see Section 2.2.3).

\begin{tabular}{ccccccc}
\hline$D / h$ & $\Delta t(\mathrm{~s})$ & $c\left(\mathrm{~m} \mathrm{~s}^{-1}\right)$ & $s_{1}$ & $s_{2}$ & $s_{4}, s_{6}$ & $s_{7}, s_{8}$ \\
\hline 12 & $4 \times 10^{-5}$ & 2.5 & 1.1 & 1 & 1.2 & $1 / 0.512$ \\
24 & $10^{-5}$ & 5 & 1.1 & 1 & 1.2 & $1 / 0.512$ \\
\hline
\end{tabular}

left (inlet) boundary, with the maximum value at the center of the cross section of the flow channel. At the opposite (outlet) boundary the fluid pressure is maintained at a constant value $p_{\text {out }}$. The fluid is modelled as incompressible and is characterised by a mass density $\rho_{\mathrm{f}}=10^{3} \mathrm{~kg} \mathrm{~m}^{-2}$ and a dynamic viscosity $\eta=10^{-3} \mathrm{~N} \mathrm{~s} \mathrm{~m}^{-1}$. A fixed circular disc with diameter $D=1.2 \mathrm{~mm}$ is located in the vicinity of the channel inlet, slightly eccentrically with respect to the longitudinal symmetry axis. Two different flow regimes are considered: a steady flow regime, at $\operatorname{Re}=20$, and an unsteady periodic flow regime, at $\operatorname{Re}=100$. The relevant definition of the Reynolds number is $\operatorname{Re}=\rho_{\mathrm{f}} \bar{u}_{\text {in }} D / \eta$, where $\bar{u}_{\text {in }}$ is the average value of the norm of the macroscopic fluid velocity at the channel inlet.

The parameters in Table 1 refer to numerical simulations of the boundary value problem performed with the LBM algorithm implemented in the Authors' in-house code, following the MRT approach. Two candidate spatial resolutions were tested, characterised as two different discretisation of the disc diameter, namely for $D / h=12$ and 24. In the same table, the parameters $h, \Delta t, s_{7}$ and $s_{8}$ are constrained by (20), and the remaining eigenvalues of the relaxation matrix were set following Du et al. (2006). The plots in Figure 6(b) $(\operatorname{Re}=20)$ and $6(\mathrm{c})(\operatorname{Re}=100)$ illustrate the results obtained with the highest spatial resolution.

Measurements of the drag force $F_{\mathrm{D}}$ and of the lift force $F_{\mathrm{L}}$ were performed in the simulations characterised in Table 1, for comparison with a benchmark of numerical methods of computational fluid dynamics (Schäfer, Turek, Durst, Krause, \& Rannacher, 1996). The comparison is shown in Table 2, in terms of the non-dimensional drag coefficient $C_{\mathrm{D}}=2 F_{\mathrm{D}} /\left(\rho_{\mathrm{f}} \bar{u}_{\text {in }}^{2} D\right)$ and lift coefficient $C_{\mathrm{L}}=2 F_{\mathrm{L}} /\left(\rho_{\mathrm{f}} \bar{u}_{\text {in }}^{2} D\right)$. The benchmark involved several work groups using different discretisation schemes for the Navier-Stokes equations (including finite elements, finite volumes and finite differences) and only one group using a standard implementation of LBM (cf. the MRT approach used herein). 
(a)

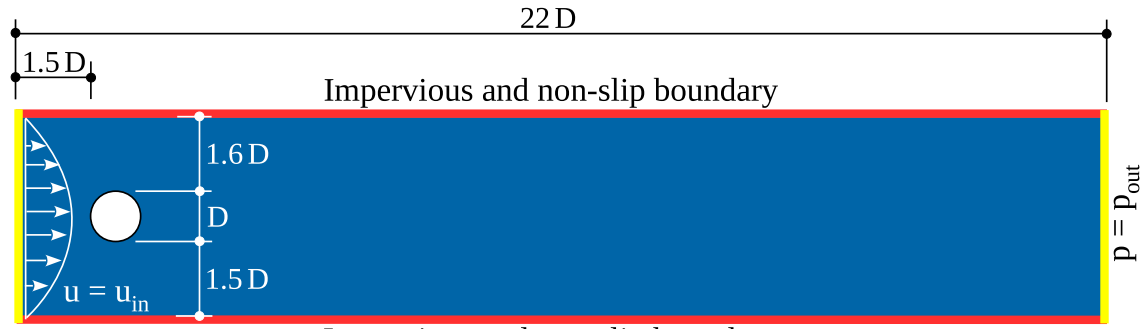

Impervious and non-slip boundary

(b)

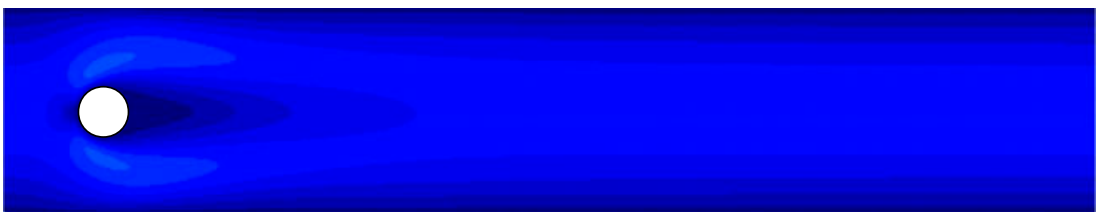

(c)

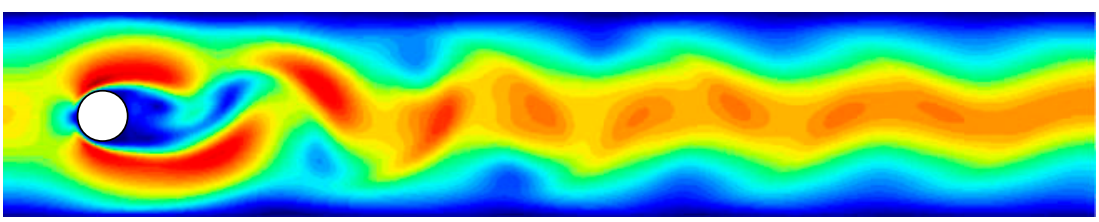

0

Fluid velocity $\left[\mathrm{m} \mathrm{s}^{-1}\right]$

0.2

Figure 6. Simulations of an incompressible fluid past a fixed circular disc of diameter $D=1.2 \mathrm{~mm}$ : (a) geometric configuration and boundary conditions; (b) norm of the macroscopic fluid velocity at $\operatorname{Re}=20$, after the flow has entered steady state; (c) norm of the macroscopic fluid velocity at $\mathrm{Re}=100$, for a state of maximum drag force, after the flow has entered the unsteady periodic regime. The results represented in (b) and (c) were obtained with the highest spatial resolution in Table 1.

The values obtained in Schäfer et al. (1996) from continuum-based methods are reported in Table 2 as statistics of the most refined simulations proposed by each group in the benchmark. The results obtained with the Authors' code, for the highest spatial resolution, are consistent with the values reported for the continuum-based methods; a less satisfactory agreement is obtained, as expected, for the lowest spatial resolution, particularly as for the value of the drag coefficient in the unsteady periodic regime, which is about $8 \%$ larger than the maximum value reported for continuum-based methods.

The measures referred to in Schäfer et al. (1996) as obtained from a standard implementation of LBM are singled out at the end of the table. The relevant values of the drag and lift coefficients for the unsteady regime are significantly higher than those referred to continuum-based methods or obtained with the Authors' code. However, the information provided in Schäfer et al. (1996) does not enable to state whether this discrepancy is due to a low spatial resolution or to other features or parameters of the relevant simulation.

In conclusion, the comparison in Table 2 shows that for the parameters referred in Table 1 to the ratio $D / h=24$, the LBM algorithm implemented in the Authors' code provides an accurate evaluation of the drag and lift forces for the configuration in Figure 6(a), up to $\operatorname{Re}=100$. 
Table 2. Calculated drag $\left(C_{\mathrm{D}}\right)$ and lift $\left(C_{\mathrm{L}}\right)$ coefficients for the problem in Figure 6(a): comparison between the values obtained with the Authors' code (using the parameters in Table 1) and a compilation of the results from the benchmark in Schäfer et al. (1996). The values for $\mathrm{Re}=20$ are measured at steady state. Those for $\mathrm{Re}=100$ are measured as maxima over one fluctuation period, after the flow has entered the unsteady periodic regime.

\begin{tabular}{|c|c|c|c|c|c|}
\hline \multirow[b]{2}{*}{ Numerical method } & \multirow[b]{2}{*}{ Reference } & \multicolumn{2}{|c|}{$\operatorname{Re}=20$} & \multicolumn{2}{|c|}{$\operatorname{Re}=100$} \\
\hline & & $C_{\mathrm{D}}$ & $C_{\mathrm{L}}$ & $C_{\mathrm{D}}$ & $C_{\mathrm{L}}$ \\
\hline LBM-MRT $(D / h=12)$ & Present & 5.7282 & 0.0112 & 3.5223 & 1.0012 \\
\hline LBM-MRT $(D / h=24)$ & Present & 5.6175 & 0.0106 & 3.2195 & 0.9109 \\
\hline Continuum-based (min.) & Schäfer et al. (1996) & 5.5069 & 0.0105 & 3.0804 & 0.7256 \\
\hline Continuum-based (avg.) & Schäfer et al. (1996) & 5.5770 & 0.0136 & 3.2077 & 0.9686 \\
\hline Continuum-based (max.) & Schäfer et al. (1996) & 5.6323 & 0.0329 & 3.2460 & 1.0692 \\
\hline Standard LBM & Schäfer et al. (1996) & 5.8190 & 0.0110 & 4.1210 & 1.6120 \\
\hline
\end{tabular}

\subsection{Coupling scheme}

\subsubsection{Hydrodynamic forces on the grains}

The actions exchanged between the fluid and the grains are computed by the LBM algorithm and summed, in the DEM algorithm, to the resultant forces and force moments acting on each grain due to contact interactions. The computation of the hydrodynamic actions on a single grain can be illustrated with reference to Figure 4, in which the curved fluid-solid boundary can be assumed to represent the profile of a grain. The action on the grain at $\mathbf{x}_{\mathrm{b}}$ is computed as the momentum transferred by the fluid node $\mathbf{x}_{\mathbf{f}}$ per unit time (Ladd, 1994a), ${ }^{5}$ i.e. as

$$
\mathbf{f}\left(\mathbf{x}_{\mathrm{b}}\right)=\frac{m h^{2}}{\Delta t}\left(f_{8}^{\mathrm{c}}\left(\mathbf{x}_{\mathrm{f}}, t\right) \mathbf{c}_{8}-f_{6}\left(\mathbf{x}_{\mathrm{f}}, t+\Delta t\right) \mathbf{c}_{6}\right)
$$

and can be generalised for arbitrary boundary nodes (cf. Section 2.2.4):

$$
\mathbf{f}\left(\mathbf{x}_{\mathrm{b}}\right)=\frac{m h^{2}}{\Delta t}\left(f_{\hat{\alpha}}^{\mathrm{c}}\left(\mathbf{x}_{\mathrm{f}}, t\right) \mathbf{c}_{\hat{\alpha}}-f_{\alpha}\left(\mathbf{x}_{\mathrm{f}}, t+\Delta\right) \mathbf{c}_{\alpha}\right)
$$

The resultant hydrodynamic force is computed by summation of (24) over the nodes $\mathbf{x}_{\mathrm{b}}$ encountered along the boundary of the grain. The analogous sum for the cross products $\left(\mathbf{x}_{\mathrm{b}}-\mathbf{x}_{\mathrm{g}}\right) \times \mathbf{f}\left(\mathbf{x}_{\mathrm{b}}\right)$ provides the resultant hydrodynamic force moment at the mass centre $\mathbf{x}_{\mathrm{g}}$ of the grain.

\subsubsection{Hydraulic radius}

A permeable pore network cannot result in 2D from a connected contact network as developed by compacted granular assemblies, for geometrical reasons. To overcome this limitation, the hydraulic radius $r_{\mathrm{h}}$ of the grain in the LBM model is set to a smaller value than the reference radius $r$ of the DEM model. Permeabilities and drag forces of realistic orders of magnitude (i.e. as expected for 3D assemblies with equivalent grain size distributions) are enabled for assemblies with millimetric grains by ratios $r_{\mathrm{h}} / r$ about 0.8 (Boutt, Cook, McPherson, \& Williams, 2007; Boutt, Cook, \& Williams, 2011; Cui et al., 2012).

A related issue is that of the minimal size of the fluid gap between two grains, in the LBM model, as illustrated in Figure 7. For numerical stability, and consistently with the numerical 


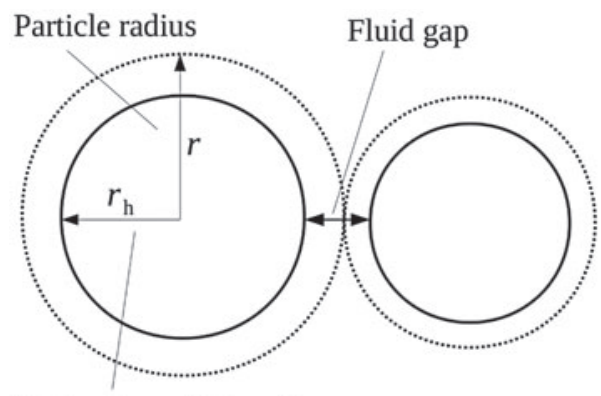

Hydraulic particle radius

Figure 7. Illustration of the hydraulic radius.

scheme for the representation of no-slip moving boundaries illustrated in Section 2.2.4, the fluid gap should always allow at least two arrays of parallel lattice nodes, i.e.

$$
r-r_{\mathrm{h}}>\sqrt{2} h+\frac{\left(\Delta x_{\mathrm{n}}\right)_{\max }}{2}
$$

in which $\left(\Delta x_{\mathrm{n}}\right)_{\max }$ is the maximum expected overlap between grains in the DEM model and the factor $\sqrt{2}$ refers to the least favourable scenario (i.e. when the centres of the two grains are aligned along a diagonal direction of the lattice).

An additional requirement concerns the resolution, in terms of lattice nodes, of the hydraulic radius. As discussed by Yu et al. (2003),

$$
\frac{r_{\mathrm{h}}}{h}>5
$$

is required for reliable estimates of the hydrodynamic actions on the grains at lowto-moderate Reynolds numbers (namely for $10<\mathrm{Re}<100$, where the Reynolds number Re herein refers to the hydraulic radius and the representative fluid velocity in the pore network).

A numerical issue of relevance in the coupling between DEM and LBM is the significant difference between the optimal time steps for the two algorithms. The time step $\Delta t$ of the LBM algorithm is a compromise between several requirements; it is related to the dynamic viscosity through (20), or through the second equation in (15) for the single relaxation time model; it enters the definition of the computational Mach number, that can be expressed as $\mathrm{Ma}=\|\mathbf{u}\| \Delta t / h$ and should be kept small enough compared with unity for reasons that are apparent in Section 2.2.1 (a limit value of 0.1 for Ma is a compromise choice often encountered in the literature). The time step $\delta t$ of the DEM algorithm is usually set to a fraction of the oscillation period for a representative pair of grains:

$$
\delta t=\alpha \sqrt{\frac{\pi \bar{r}^{2} \rho_{\mathrm{s}}}{k_{\mathrm{n}}}}
$$

where $\rho_{\mathrm{S}}$ is the mass density of the grains, $\bar{r}$ is the average radius in the assembly and $\alpha$ is of the order of $10^{-2}$. Since in general $\delta t<\Delta t$, the DEM computation cycles are subcycles of the LBM cycles, and $\Delta t$ is set to a multiple of $\delta t$. 
(a) DEM

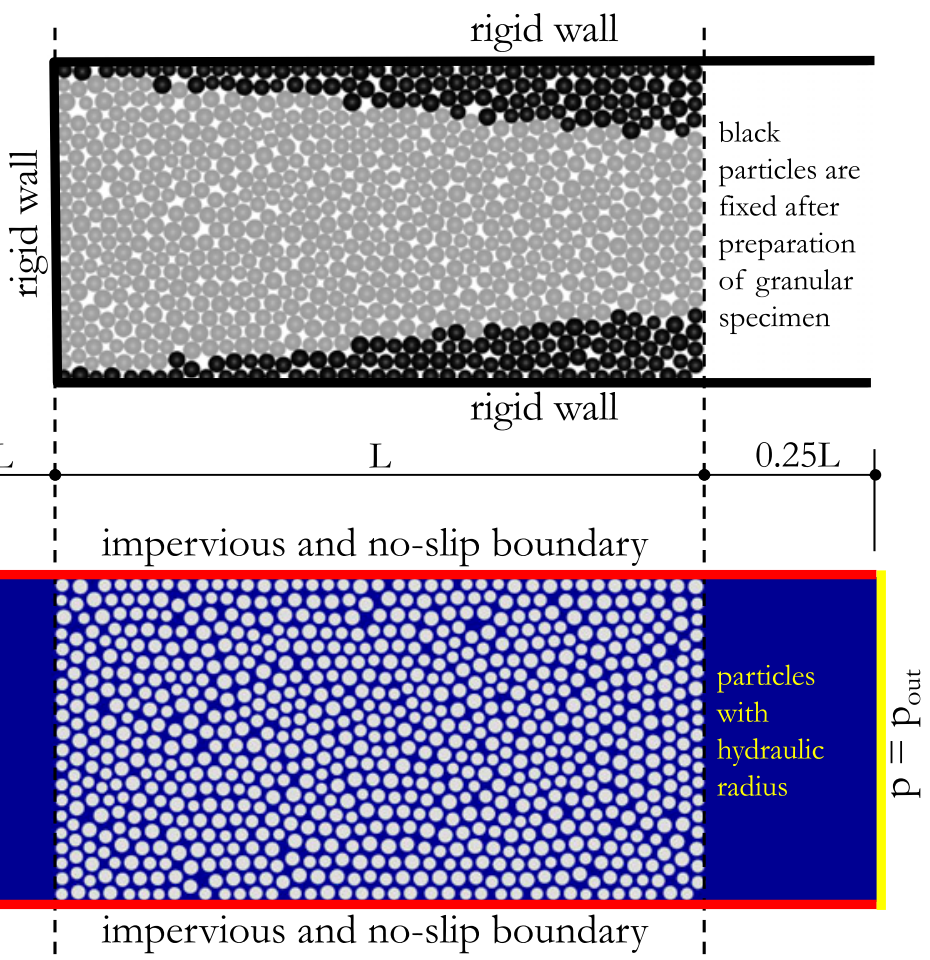

Figure 8. Simulation of backward erosion: geometric configurations ( $L=66.8 \mathrm{~mm}, H=33 \mathrm{~mm}$ ), boundary conditions and initial particle arrangements for the coupled models developed with DEM (a) and LBM (b).

\section{Backward erosion test}

\subsection{Preparation of the granular specimen}

The arrangement in Figure 8(a) represents the numerical specimen employed to model a granular soil REV located on the upstream side of the pipe face. It consists of 800 circular grains with mass density $\rho_{s}=2.65 \times 10^{3} \mathrm{~kg} \mathrm{~m}^{-2}$ and radii randomly dispersed in the range from 0.75 to $0.95 \mathrm{~mm}^{6}$

The specimen was obtained, with the numerical implementation of DEM described in Section 2.1, by the procedure reported here below which did not involve fluid-solid coupling. A preliminary "lubricated" isotropic compaction from an initial, randomly dispersed configuration was performed by convergence of four rigid walls parallel to the horizontal and vertical directions in the figure. At the end of compaction, after the confining pressure had reached approximately $30 \mathrm{kN} \mathrm{m}^{-1}$, the walls were maintained at fixed positions till the grains reached a state of near static equilibrium. All the existing contacts were then converted to bonds, with the exception of those at the right wall. This wall was then carefully removed (i.e. slowly translated to the right until all contacts on it were lost) while the left, top and bottom walls were being hold fixed. From null to low-enough velocities were assigned to the confining walls for the entire procedure to be performed under quasi-static conditions.

The values of the contact parameters during the above procedure are listed in Table 3. The normal contact stiffness $k_{\mathrm{n}}$ was set according to an expected order of magnitude of 
Table 3. Contact parameters employed during preparation of the specimen.

\begin{tabular}{lccccc}
\hline & & \multicolumn{2}{c}{ Initialisation of the bonds } & \\
\cline { 3 - 5 } & & & \multicolumn{2}{c}{ Grain-to-wall } & \\
\cline { 3 - 5 } & During compaction & Grain-to-grain & Right wall & Other walls & After the initialisation \\
\hline$k_{\mathrm{n}}\left(\mathrm{N} \mathrm{m}^{-1}\right)$ & $5.4 \times 10^{7}$ & $5.4 \times 10^{7}$ & $5.4 \times 10^{7}$ & $5.4 \times 10^{7}$ & $5.4 \times 10^{7}$ \\
$k_{\mathrm{t}}\left(\mathrm{N} \mathrm{m}^{-1}\right)$ & $5.4 \times 10^{7}$ & $5.4 \times 10^{7}$ & $5.4 \times 10^{7}$ & $5.4 \times 10^{7}$ & $5.4 \times 10^{7}$ \\
$c_{\mathrm{n}}\left(\mathrm{N} \mathrm{s} \mathrm{m}^{-1}\right)$ & 500 & 500 & 500 & 500 & 500 \\
$c_{\mathrm{t}}\left(\mathrm{N} \mathrm{s} \mathrm{m}^{-1}\right)$ & 500 & 500 & 500 & 500 & 500 \\
$A(\mathrm{~N})$ & 0 & 1 & 0 & $10^{6}$ & 0 \\
$C(\mathrm{~N})$ & 0 & 4 & 0 & $10^{6}$ & 0 \\
$\mu$ & 0 & 0.5 & 0.5 & 0.5 & 0.5 \\
\hline
\end{tabular}

$10^{-4}$ diameter values for the contact deflection at the target confining pressure. ${ }^{7}$ Due to lack of specific information, the tangential contact stiffness $k_{\mathrm{t}}$ was set as the same value as the normal stiffness. In order to let the system dissipate efficiently, the normal and tangential damping coefficients $c_{\mathrm{n}}$ and $c_{\mathrm{t}}$ were set at about $80 \%$ of the critical value for harmonic oscillators (normal and tangential, resp.) consisting of two interacting discs with average diameters. The friction coefficient $\mu$ was chosen as an arbitrary, yet sensible value. However, during compaction, lubricated conditions were prescribed via null values of the latter parameter and of the contact adhesion and cohesion, $A$ and $C$, for both grain-to-grain and grain-to-wall contacts, in order to obtain a dense and highly textured configuration. The contacts detected at the end of compaction, after relaxation of the specimen, were then "initialised" as reported in the same table. Namely, grain-to-grain contacts were converted to viscoelastic bonds. The relevant value of contact adhesion is consistent with an extremely weak macroscopic tensile traction, with order of magnitude $10^{2} \mathrm{~N} \mathrm{~m}^{-1}$, and the contact cohesion was set as a multiple of the contact adhesion. The same holds for grain-to-wall contacts at the left, top and bottom walls, but with extremely large (actually unattainable) values of adhesion and cohesion, in order to create monolayers of grains sharing permanent bonds with the walls. At the right wall, grain-to-wall contacts were not initialised as bonds, but kept as unilateral lubricated viscoelastic contacts for minimal disturbance of the specimen during the removal of the wall. New contacts created after the initialisations of the bonds are considered by default as unilateral frictional-viscoelastic. Finally, the time step $\delta t$ of the DEM algorithm was set to $2.5 \cdot 10^{-7} \mathrm{~s}$ consistently with (25).

By the time the right wall had been completely removed, the stress on the left, top and bottom walls relaxed to negligible values. Meanwhile the bond-related coordination number (i.e. the average number of grain-to-grain bonds per grain) was reducing from 2.805 to 1.383 . Furthermore, after removal of the right wall, the overall population of grainto-grain contacts consisted almost entirely of bonds (unilateral contacts were less than $1 \%$ ). The polar histograms in Figure 9 represent the angular distributions of grain-to-grain contacts at the initialisation of the bonds and after removal of the right wall. The distributions refer to the whole population of grain-to-grain bonds, as well as separately to compressive contacts (bonds and unilateral contacts) or tensile bonds. The inherent anisotropy of the distributions in the same figure is inherited from the compaction procedure, due to a number of factors such as limited range of grain diameters, the shape and size of the specimen and the effect of rigid walls. 


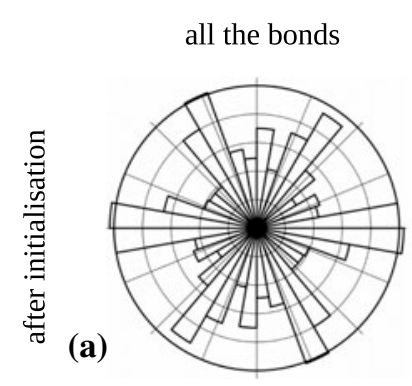

$$
\begin{aligned}
& \text { compressive bonds } \\
& \text { and contacts }
\end{aligned}
$$

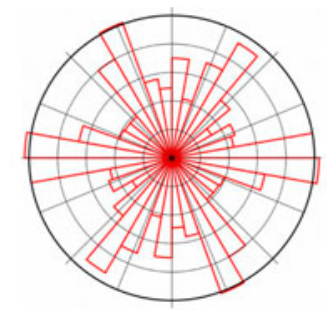

tensile bonds
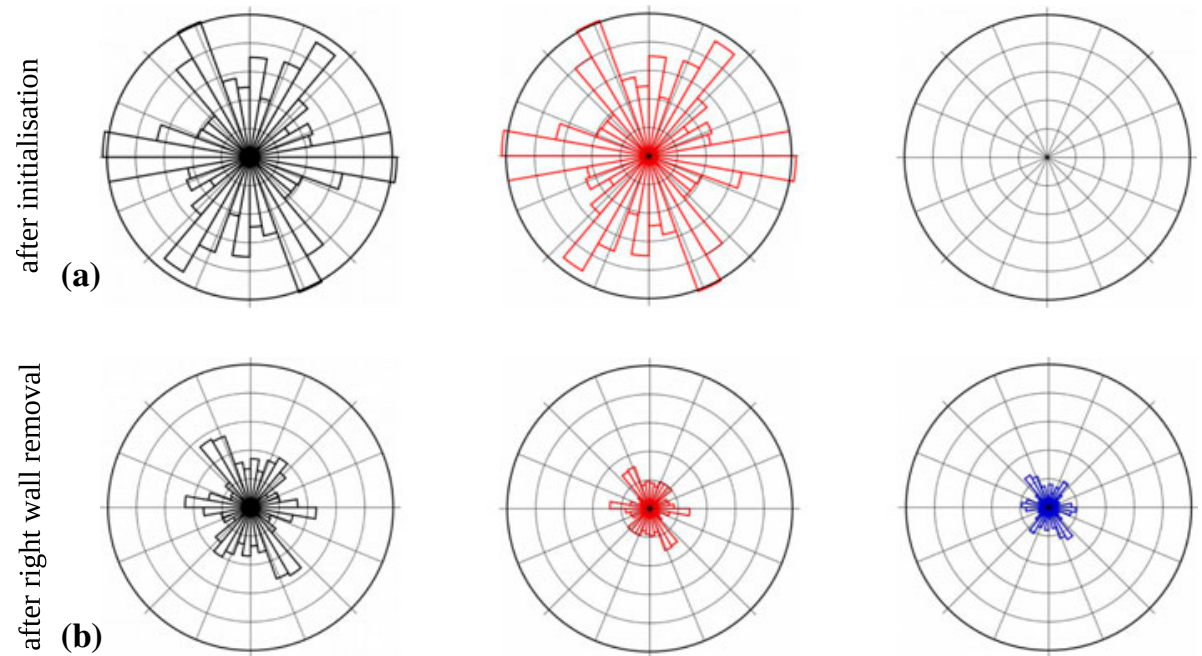

Figure 9. Specimen preparation: angular distributions of grain-to-grain contacts after initialisation (a) and right wall removal (b). The histograms refer to the whole populations of bonds (left), or to the populations of compressive contacts (bonds and unilateral contacts, center) and tensile bonds (right). The outer circle in each plot refers to 0.25 coordination number units per angular sector bar (on 18 angular sector bars per $\pi$ radians). The angle of a grain-to-grain contact is given by the orientation of the unit vector $\mathbf{n}$ in Figure 1.

\subsection{Flow-induced erosion of the granular specimen}

The coupling of DEM and LBM presented in Section 2 was employed to model the backward erosion induced by an interstitial fluid flow in the specimen obtained from the "dry" preparation procedure described in the previous section. The simulation is designed with reference to the physical situation encountered by a REV at the soil-pipe interface in the front region, eroded by a water flow exiting the REV across its unconfined face (i.e. the right face in Figure 8(a)).

With regard to the DEM model, during the erosion test the specimen resulting from the preparation procedure was confined by a rigid wall on the left, maintained at the same position as at the end of the preparation procedure, while the degrees of freedom of the blackcoloured grains in Figure 8(a) were locked so as to form two oblique rough boundaries at the top and the bottom, respectively. As illustrated in Figure 10, the resulting trapezoidal shape of the REV region, consisting of mobile (gray-coloured) grains, is reminiscent of an angular sector at the supposedly curved soil-pipe interface in the front region.

How the presence of the ambient fluid affects the contact behaviour is still to be assessed and more advanced, dissipative contact models are being proposed that include fluid properties (Nguyen \& Ladd, 2003; Tomac \& Guierrez, 2013; Yang \& Hunt, 2008). However, a refinement in this sense of the contact model is expected to improve significantly the quality of the results only when the behaviour of a well-identified granular material is among the objectives of the numerical simulation, which is not the case in this study. Hence, the same contact model and parameters as at the end of the preparation procedure were used for modelling the backward-erosion process. 


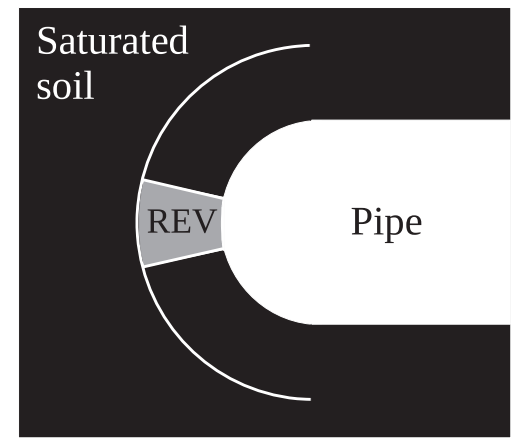

Figure 10. Sketch of the granular REV as part of the soil-pipe interface in the front region. The REV is confined on the upstream (left) side by the soil in the intact region and laterally by the contiguous interface elements.

With regard to the LBM model, Figure 8(b) represents the hydraulic boundary conditions implemented in the simulation, as well as the "hydraulic configuration" of the specimen at the beginning of the test: this is the same configuration as in Figure 8(a), but after reduction in the radii by a factor of 0.8 , according to the hydraulic radius hypothesis (see Section 2.3.2). An incompressible fluid flow, confined by the impervious walls located at the top and the bottom of the specimen, was enforced through the REV by the pressure difference

$$
\Delta p=p_{\text {out }}-p_{\text {in }}<0
$$

between the right (outlet) and the left (inlet) boundaries of the fluid domain. As in Section 2.2.6, the fluid is characterised by a mass density $\rho_{\mathrm{f}}=10^{3} \mathrm{~kg} \mathrm{~m}^{-2}$ and a dynamic viscosity $\eta=10^{-3} \mathrm{~N} \mathrm{~s} \mathrm{~m}^{-1}$, which are rough estimates for the properties of water at $20^{\circ} \mathrm{C}$. The evolution of the normalised pressure difference $-\Delta p /\left(\rho_{\mathrm{f}} g L\right)$, where $g$ is the standard gravity acceleration, is plotted in Figure 11(i) for the whole duration of the test. Consistent with (23), the pressure values $p_{\text {out }}$ and $p_{\text {in }}$ are actually assigned in terms of mass densities, i.e. $p_{\text {out }}=c_{s}^{2} \rho_{\mathrm{f}}$ and $p_{\text {in }}=c_{\mathrm{s}}^{2}\left(\rho_{\mathrm{f}}+\Delta \rho\right)$, see (15). The flow being considered here as incompressible in the sense expressed in Section 2.1, the density $\rho_{\mathrm{f}}$ is to be understood as the reference mass density $\rho_{0}$ in that section and the ratio $\Delta \rho / \rho_{\mathrm{f}}$ is to be of the same or higher order than the square of the computational Mach number, consistently with (13).

At the beginning of the simulation, the distribution function at each fluid node was initialised to the values of the Maxwell distribution $f_{\alpha}^{\mathrm{eq}}$, computed for the mass density $\rho_{\mathrm{f}}$ and for $\mathbf{u}=\mathbf{0}$, where $\mathbf{u}$ is the macroscopic fluid velocity as in (12). The set of lattice parameters controlling directly or indirectly the numerical implementation of the LBM model was chosen consistently with a number of requirements posed in Sections 2.2-2.3. In particular, the spacing between neighbouring lattice nodes and the lattice time step were set as $h=5 \times 10^{-5} \mathrm{~m}$ and $\Delta t=10^{-5} \mathrm{~s}$, respectively. A first requirement on the two parameters, from (20), refers to the imposed values of dynamic viscosity and mass density of the fluid. Additionally, the choice of $h$ and $\Delta t$ determines the lattice-related sound speed $c_{\mathrm{S}}=c / \sqrt{3}=h /(\sqrt{3} \Delta t)$ which, in turn, affects the assignment of the pressure boundary condition at the inlet and the outlet of the flow conduit (see Section 2.2.5). Other requirements on the spacing $h$ between neighbouring lattice nodes can be referred to the spatial resolution for accurate estimates of the hydrodynamic actions (see Section 2.2.6) and to the hydraulic radius assumption (see Section 2.3.2). The former of the last two 

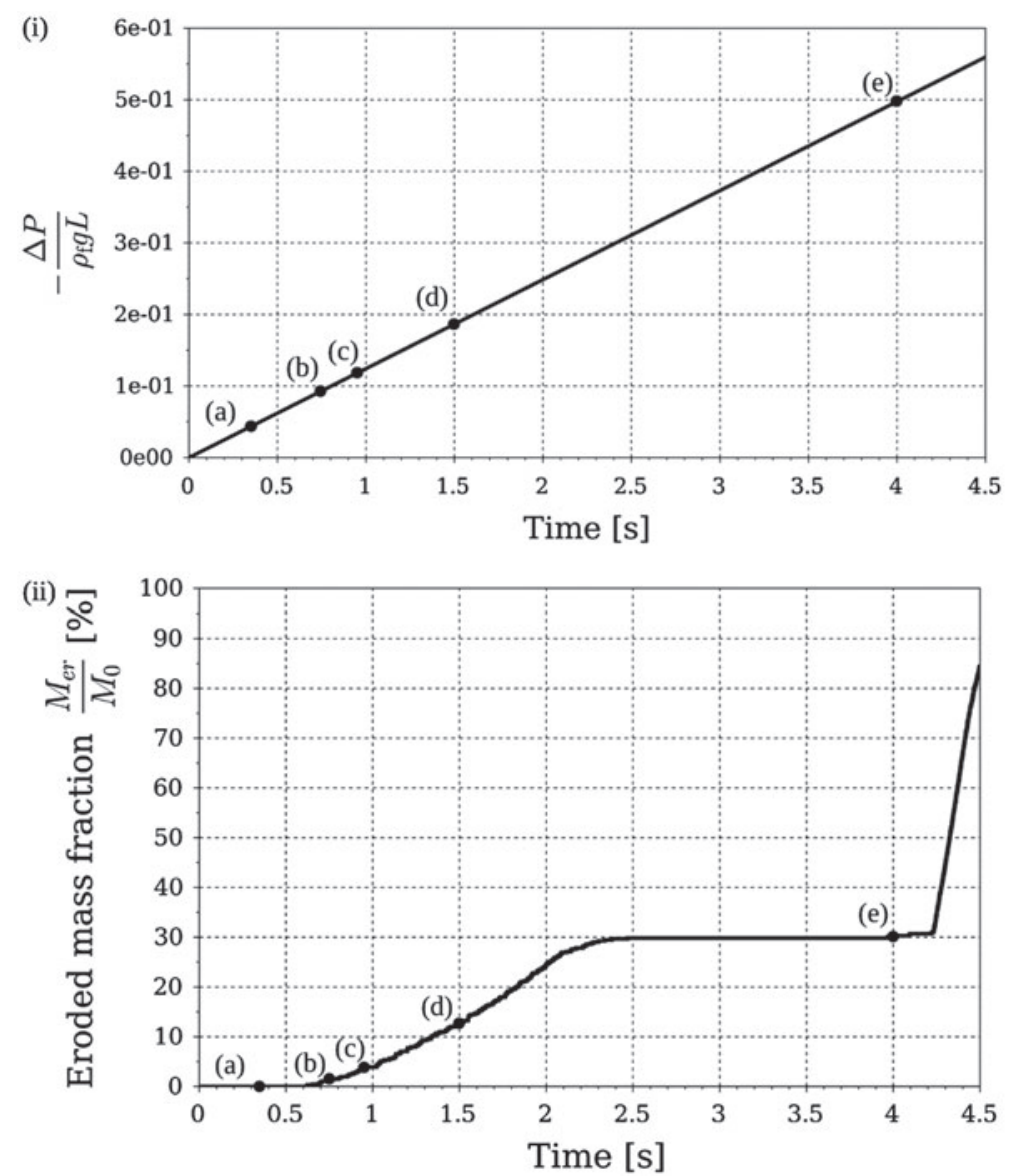

Figure 11. Evolution of the test: normalised pressure difference (i) and eroded mass fraction (ii). Labels refer to $t=0.35 \mathrm{~s}(\mathrm{a}), 0.75 \mathrm{~s} \mathrm{(b)}, 0.95 \mathrm{~s}(\mathrm{c}), 1.5 \mathrm{~s}$ (d) and $4.0 \mathrm{~s}$ (e).

requirements was the most restrictive, and $h$ was set equal to $1 / 12$ of the hydraulic radius of the smallest grain, corresponding to the ratio $D / h=24$ in Section 2.2.6. Accordingly, the eigenvalues of the relaxation matrix and the characteristic lattice speed $c$ were set as assessed in that section for the same resolution. Notice, finally, that the value of the mass $m$ of the fluid particle has strictly no effect on the simulation.

Representative Reynolds numbers for the seepage flow were estimated according to the definition $\operatorname{Re}=\rho_{\mathrm{f}} \bar{u}_{\mathrm{p}}\left(2 \bar{r}_{\mathrm{h}}\right) / \eta$, where $\bar{u}_{\mathrm{p}}$ is the average fluid velocity in the pore space and $\bar{r}_{\mathrm{h}}$ is the average hydraulic radius. The values reported in Table 4 are consistent with those considered in Section 2.2.6 for the validation of the implemented algorithm and of the relevant parameters.

As stressed in Section 2.2.4, the conservation of fluid mass is not an implicit feature of the implemented no-slip condition for moving boundaries. Therefore, the total fluid mass was recorded during the first part of the erosion test, up to $t=1.55 \mathrm{~s}$, i.e. before the first grain started exiting the fluid domain through the channel outlet. An increase of $0.63 \%$ was measured, which is considered consistent with the required accuracy. 
(a)

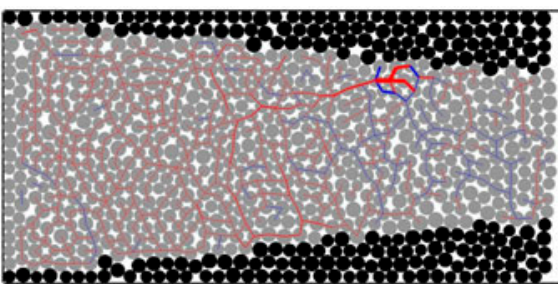

(b)

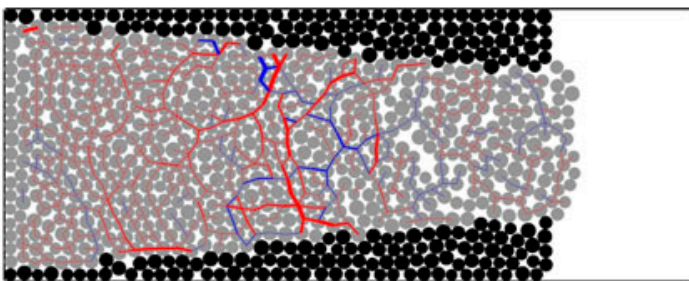

(c)

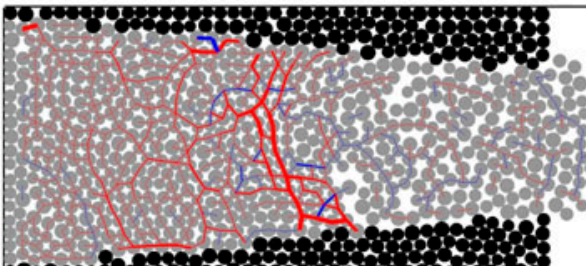

\begin{tabular}{c}
$\begin{array}{c}\text { Max. compressive } \\
\text { force } 1.27 \mathrm{~N}\end{array}$ \\
\hline $\begin{array}{l}\text { Max. tensile } \\
\text { force } 0.87 \mathrm{~N}\end{array}$ \\
\hline
\end{tabular}

(d)

force $0.87 \mathrm{~N}$

(e)

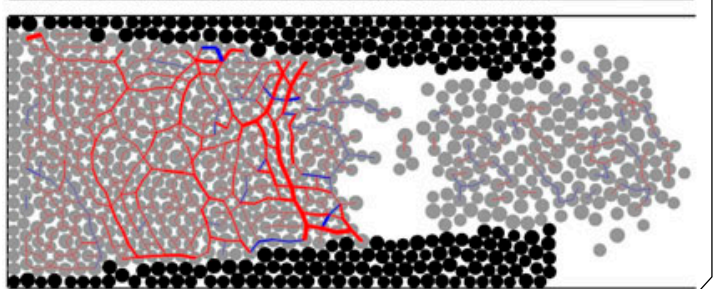

Max. compressive
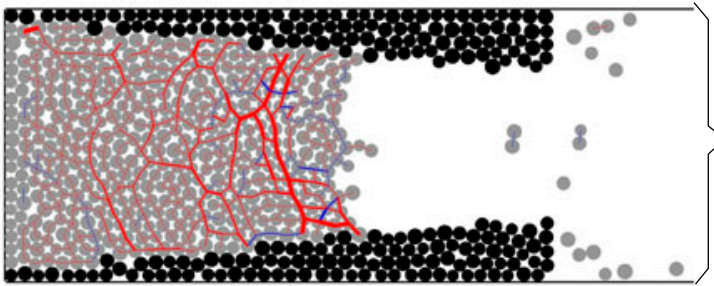

force $3.16 \mathrm{~N}$

Max. tensile force $0.99 \mathrm{~N}$

Figure 12. Evolution of the test: compressive and tensile force chains (blue-coloured and red-coloured lines, resp.) and configurations of the specimen at $t=0.35 \mathrm{~s}(\mathrm{a}), 0.75 \mathrm{~s} \mathrm{(b),} 0.95 \mathrm{~s} \mathrm{(c),} 1.5 \mathrm{~s} \mathrm{(d)}$ and $4.0 \mathrm{~s}(\mathrm{e})$.

Table 4. Values of the Reynolds number for the seepage flow at the time instants identified in Figure 11.

\begin{tabular}{llllll}
\hline$t(\mathrm{~s})$ & 0.35 & 0.75 & 0.95 & 1.5 & 4.0 \\
\hline $\operatorname{Re}$ & 7.15 & 17.81 & 21.23 & 33.62 & 74.65 \\
\hline
\end{tabular}


(a)

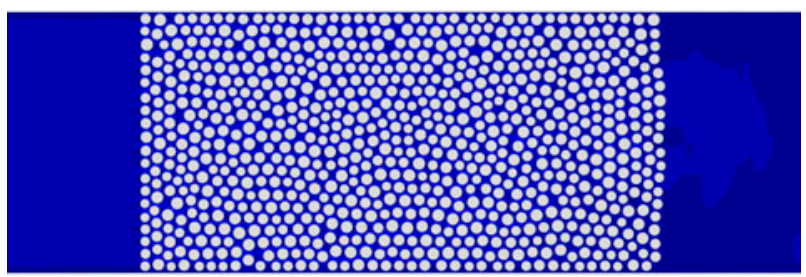

(b)

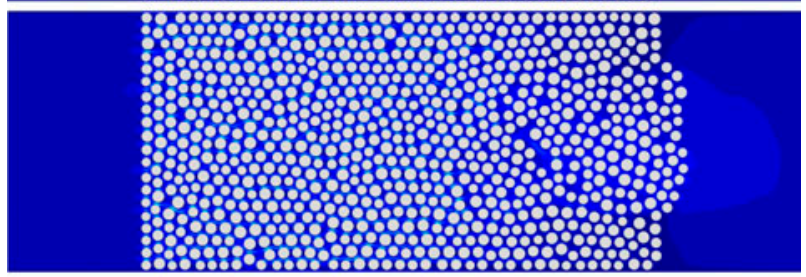

(c)
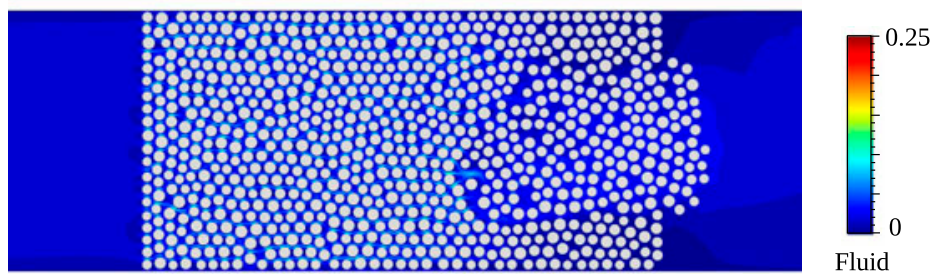

Fluid velocity

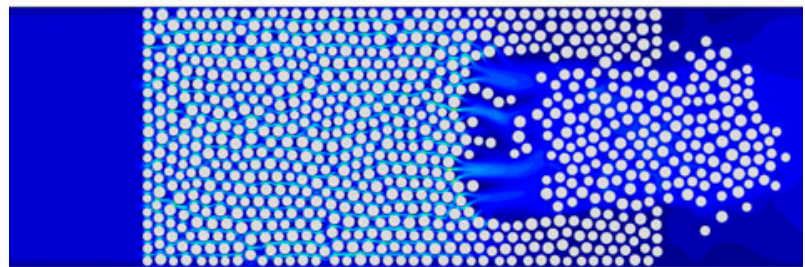

$\left[\mathrm{m} \mathrm{s}^{-1}\right]$

(d)

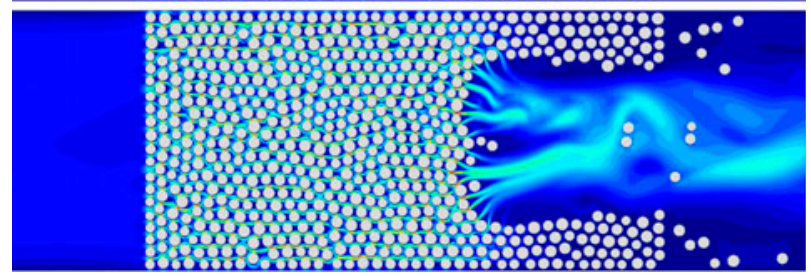

Figure 13. Evolution of the test: norm of the macroscopic fluid velocity and hydraulic configurations of the specimen at $t=0.35 \mathrm{~s} \mathrm{(a),} 0.75 \mathrm{~s}(\mathrm{~b}), 0.95 \mathrm{~s} \mathrm{(c),} 1.5 \mathrm{~s} \mathrm{(d)} \mathrm{and} 4.0 \mathrm{~s}(\mathrm{e})$.

\subsection{Observed results}

The evolution of the erosion process during the test is quantified in Figure 11(ii) in terms of the eroded mass fraction $M_{\mathrm{er}} / M_{0}$, where $M_{\mathrm{er}}$ and $M_{0}$ are the eroded and erodible mass, respectively. At a given time instant, the eroded mass is defined as the cumulative mass of the grains having crossed the right vertical limit of the initial configuration of the specimen (i.e. the dashed line in Figure 8(a)). The erodible mass is the total mass of the movable (gray-coloured) grains in Figure 8(a).

At the beginning of the test, for low-enough values of the pressure difference, no erosion was observed. Visual information on the process at this stage is provided in Figures 12(a), 13(a) and 14(a), corresponding to the marks with label (a) in Figure 11. Even at very low values, the hydraulic load affects the stress transmission through force chains within the specimen: 
(a)

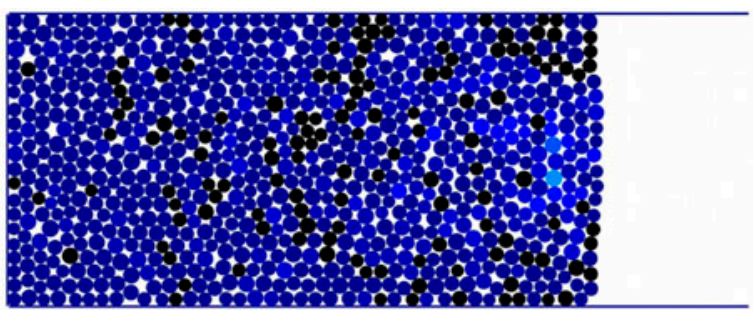

(b)

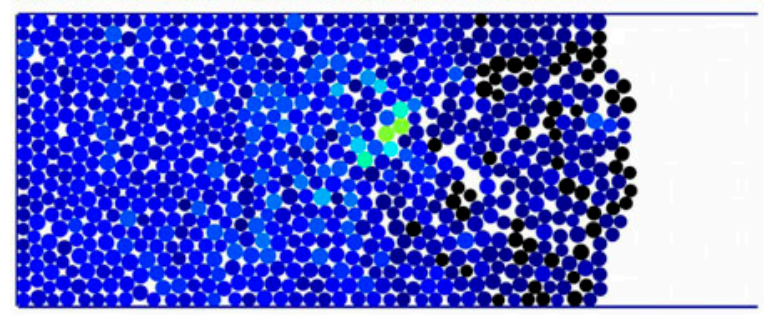

(c)
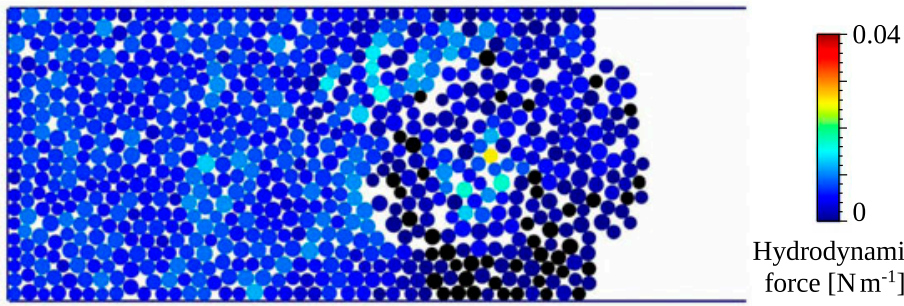

Hydrodynamic force $\left[\mathrm{N} \mathrm{m}^{-1}\right]$

(d)

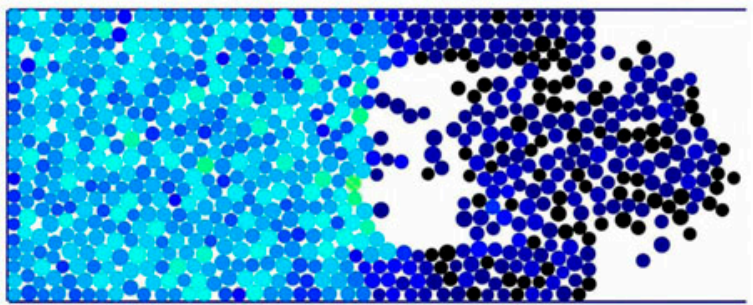

(e)

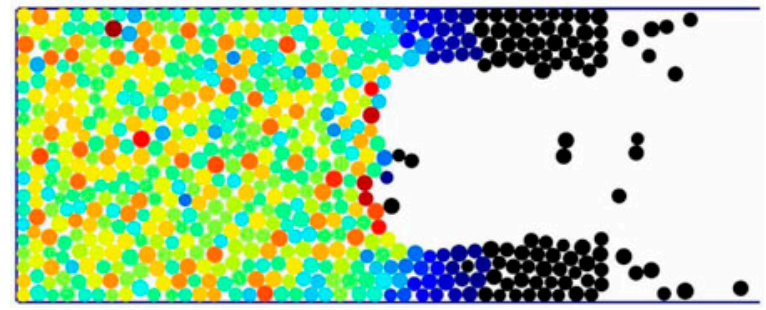

Figure 14. Evolution of the test: norm of the hydrodynamic force on each grain for the configurations of the specimen at $t=0.35 \mathrm{~s} \mathrm{(a),} 0.75 \mathrm{~s} \mathrm{(b),} 0.95 \mathrm{~s}(\mathrm{c}), 1.5 \mathrm{~s}$ (d) and $4.0 \mathrm{~s}(\mathrm{e})$.

a concentration of compressive and tensile force chains can be observed roughly at the upstream and downstream sides of the specimen, respectively. The comparison between Figures 9(b) and 15(a) suggests that the resistance mechanism opposed by the assembly to the drag forces on the grains is lead by the compressive force chains. Moreover, the contribution of unilateral contacts is not anymore negligible, compared with contact bonds.

Backward erosion was observed for larger values of the driving pressure difference, roughly in the branch from (b) to (d) in Figure 11. As shown by the corresponding config- 
(a)

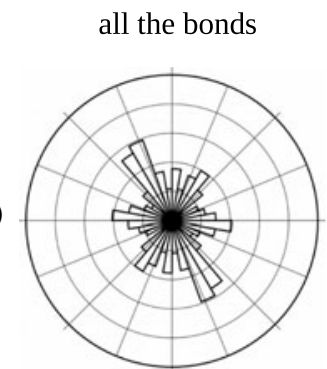

(b)

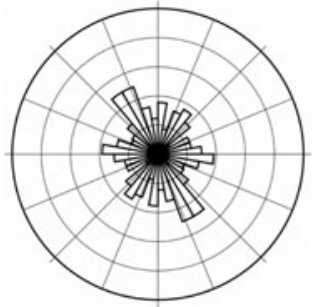

(c)

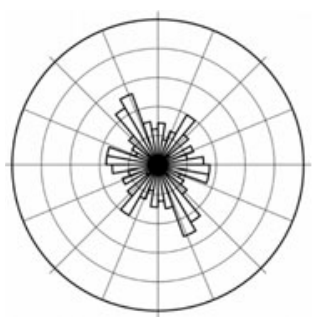

(d)

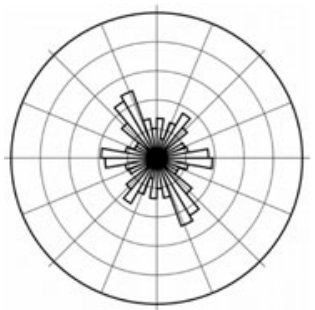

compressive bonds

and contacts
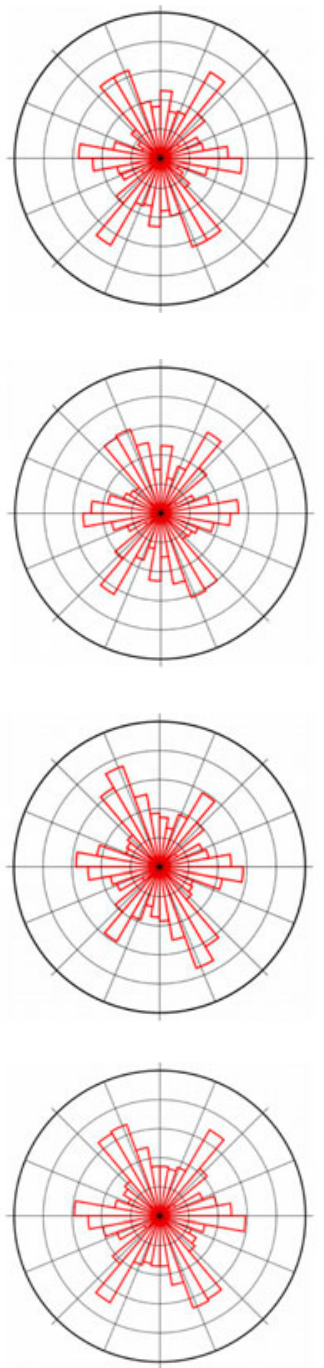

tensile bonds
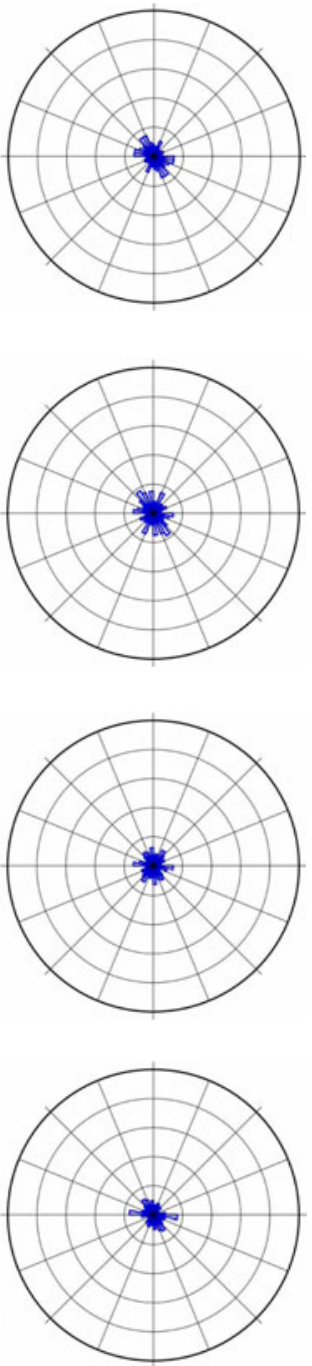

Figure 15. Evolution of the test: angular distributions of grain-to-grain contacts at $t=0.35 \mathrm{~s}$ (a), $0.75 \mathrm{~s}(\mathrm{~b}), 0.95 \mathrm{~s}(\mathrm{c}), 1.5 \mathrm{~s}$ (d). See Figure 9 for definitions and units. Only movable grains are considered (cf. Figure 9).

urations in Figures 12-14, the eroded mass is detached and transported by the flow in the form of a large, deformable cluster of grains. A main arch of force chains in the middle of the specimen can be clearly observed, separating the compressive upstream region from the downstream region being eroded.

The increasing branch after point (d) of the eroded mass diagram in Figure 11(ii) is to be referred to previously mobilised grains progressively exiting the right limit of the measure volume: no significant further detachment of granular material was observed up to point (e). This was due to the resistance provided by the persisting arch of force chains. However, 
the continuously increasing hydraulic gradient resulted in further loading of the arch until a global instability appeared after (d). The resulting fluidisation of the specimen, in which all erodible grains were washed away simultaneously, is represented by the final increasing branch in the same figure (but it is not represented in Figures 12-14).

The plots in Figure 14 illustrate the evolution of the norm of the hydrodynamic forces on the grains, during the erosion test, and refer to the same configurations identified in Figure 11. Spatial variability of the hydrodynamic forces is observed at the scale of the specimen, with a tendency for lower values towards the downstream side of the granular region. Some randomness at the scale of a few grain diameters can also be observed, especially in Figure 14(d) and (e), i.e. for larger values of the imposed hydraulic gradient. Figure 14(b)-(d) also illustrate the complexity of the interaction between the fluid and solid phases during the backward erosion process. The simple analysis suggests that an accurate description of the fluid phase is likely to be an essential ingredient of the modelling of the observed phenomenon.

A further insight into the erosion process is provided by the polar histograms in Figure 15. The population consisting of both compressive bonds and compressive unilateral contacts kept essentially constant during the whole process. The population of tensile bonds increased slightly at the onset of erosion (Figure 15(a) and (b)), during the development of the weakly tensile region at the downstream side of the specimen. The latter population reduced progressively as the erosion process went on (Figure 15(b)-(d), corresponding to a reduction of about $26 \%$ of the number of tensile bonds).

These observations are consistent with the conjecture that arching is the dominant resistance mechanism though tensile resistance contributes by regulating the process in the region being eroded. However, a thorough analysis should include the role of damage developed at the downstream eroded region due to the removal of the right wall during the preparation procedure: a similar role is expected to be played in real situations by the damage due to stress relaxation at the soil-pipe interface.

\section{Conclusions}

The numerical test presented in this study was designed to assess the effectiveness of coupling two discrete numerical methods, namely LBM and DEM, for the micromechanical investigation of piping erosion. The attention was focused on the mechanisms involved in the backward propagation of erosion, at the pipe tip, induced by the interstitial fluid flow normal to the soil-pipe interface. Hence, the study is complementary to previous investigations on piping erosion, using similar numerical tools, but focused on the mechanism of tangential erosion at the pipe walls leading to conduit enlargement (Lominé et al., 2013; Sibille et al., 2015).

The coupled scheme discussed in Section 2 and the testing procedure presented in Section 3 enabled to observe several interesting features in relation to the targeted phenomenon. Among the latter, the most distinct effect was the marked arching through force chains, as a self-organised response of the contact/bond network to drag forces and couples applied on the grains. Further analyses are required to clearly identify the additional role of tensile traction and damage at the soil-pipe interface.

Particular care was required for the design of the test, in order to obtain realistic solutions and to ensure numerical stability. This was made possible by the accurate choice of the numerical parameters, according to separate as well as relative criteria for the two numerical schemes and consistently with a validation of the computation of the hydrodynamic actions. 
The improved numerical stability of the MRT approach enabled the required flexibility in the choice of the LBM parameters.

Further developments, currently in progress, include the use of the DEM-LBM coupling to investigate the effectiveness of available laws for erosion kinetics, for their application in the analysis of internal erosion at the scale of hydraulic structures (Rotunno et al., in preparation; Zhang, Wong, Leo, \& Bui, 2013).

\section{Disclosure statement}

No potential conflict of interest was reported by the authors.

\section{Funding}

This research was supported by GIS VOR 2012,LTDS 2012 and PRIN 2010-2011 (2010BFXRHS_004) projects as well as by one $\mathrm{PhD}$ fellowship funded by the French Ministry of National Education, Higher Education and Research. The research also benefited from several invitations of C. Callari at École Centrale de Lyon - LTDS.

\section{Notes}

1. The term on the r.h.s. of (14) is identified in He and Luo (1997b) as $\left(\rho_{0} c_{\mathrm{s}}^{2}\right)^{-1} \partial p / \partial t$. Incompressibility is thus enforced in the case of steady flow. As discussed in the same reference, ensuring incompressibility for unsteady flows implies an additional control on the temporal variations in the driving pressure.

2. The thermal energy density $e$ and the heat flux vector $\mathbf{q}$ can be identified (up to terms of the order $O\left(M^{3}\right)$ as $M \rightarrow 0$ ) as the velocity moments $e=\frac{m}{2} \sum_{\alpha=0}^{8} f_{\alpha}\left\|\mathbf{c}_{\alpha}\right\|^{2}$ and $\mathbf{q}=\frac{m}{2} \sum_{\alpha=0}^{8} f_{\alpha}\left\|\mathbf{c}_{\alpha}\right\|^{2} \mathbf{c}_{\alpha}$.

3. For a review of different models for solid boundary, including their efficiency in terms of numerical stability, the reader is referred to Yu, Mei, Luo, and Shyy (2003).

4. A different approach can be found, which is based on the extrapolation of the distribution function from the values assumed at the neighbouring fluid nodes (Lallemand \& Luo, 2003).

5. The so-called stress integration approach can be used as an alternative to the momentum approach, considered here, to compute the hydrodynamic actions on the grains. According to Yu et al. (2003), the former approach provides less consistent estimates.

6. Consistent with the reduction to a two-dimensional geometry, volume and surface densities are represented as surface and linear densities, respectively.

7. Estimates of the contact deflection under a given confining pressure can be inferred from the local elastic properties and the characteristic grain size (Roux \& Chevoir, 2005). The expected values for an assembly of quasi-monodisperse silica spheres, at $30 \mathrm{kPa}$, are of the order of $10^{-5}$ diameter values according to Hertz contact model. However, a more realistic estimate should take into account those imperfections likely to affect the contact behaviour of a real granular material. Hence, the normal contact stiffness in Table 3 are consistent with values of contact deflection of the order of $10^{-4}$ diameter values at $30 \mathrm{kNm}^{-1}$.

\section{References}

Aidun, C., Lu, Y., \& Ding, E.-J. (1998). Direct analysis of particulate suspensions with inertia using the discrete Boltzmann equation. Journal of Fluid Mechanics, 373, 287-311.

Bhatnagar, P. L., Gross, E. P., \& Krook, M. (1954). A model for collision processes in gases. I. Small amplitude processes in charged and neutral one-component systems. Physical Review, 94 , $511-525$.

Bonelli, S. (Ed.). (2013). Erosion in geomechanics applied to dams and levees. Hoboken, NJ: WileyISTE.

Bonelli, S., \& Brivois, O. (2008). The scaling law in the hole erosion test with a constant pressure drop. International Journal for numerical and analytical methods in geomechanics, 32, 1573-1595. 
Boutt, D. F., Cook, B. K., McPherson, B. J. O. L., \& Williams, J. R. (2007). Direct simulation of fluid-solid mechanics in porous media using the discrete element and lattice-Boltzmann methods. Journal of Geophysical Research, 112, B10209 (13 pages).

Boutt, D. F., Cook, B. K., \& Williams, J. R. (2011). A coupled fluid-solid model for problems in geomechanics: Application to sand production. International Journal for Numerical and Analytical Methods in Geomechanics, 35, 997-1018.

Bouzidi, M., Firdaouss, M., \& Lallemand, P. (2001). Momentum transfer of a Boltzmann-lattice fluid with boundaries. Physics of Fluids, 13, 3452-3459.

Cambou, B., Jean, M., \& Radjai, F. (Eds.). (2009). Micromechanics of granular materials. Hoboken, NJ: Wiley-ISTE.

Chareyre, B., Cortis, A., Catalano, E., \& Barthélemy, E. (2011). Pore-scale modeling of viscous flow and induced forces in dense sphere packings. Transport in Porous Media, 92, 473-493.

Cheng, L., Draper, S., \& An, H. (Eds.). (2015). Scour and erosion. Leiden: CRC Press.

Cottereau, R., Díez, P., \& Huerta, A. (2010). Modeling, with a unified level-set representation, of the expansion of a hollow in the ground under different physical phenomena. Computational Mechanics, 46, 315-327.

Cui, X., Li, J., Chan, A., \& Chapman, D. (2012). A 2D DEM-LBM study on soil behaviour due to locally injected fluid. Particuology, 10, 242-252. Advances in characterization and modeling of particulate processes.

Cundall, P. A., \& Strack, O. D. L. (1979). A discrete numerical model for granular assemblies. Géotechnique, 29, 47-65.

da Silveira, A. F. (1984). Statistical analysis of deteriorations and failures of dams. In J. L. Serafim (Ed.), Safety of Dams (pp. 55-60). Rotterdam: Balkema.

d'Humière, D. (1992). Generalized lattice Boltzmann equations. In D. Shizgal \& D. P. Weaver (Eds.), Rarefied Gas Dynamics: Theory and Simulations. Washington, DC: American Institute of Aeronautics and Astronautics.

d'Humière, D., Ginzburg, I., Krafczyk, M., Lallemand, P., \& Luo, L. -S. (2002). Multiple-relaxationtime lattice Boltzmann models in three dimensions. Philosophical Transactions of the Royal Society of London A: Mathematical, Physical and Engineering Sciences, 360, 437-451.

Du, R., Shi, B., \& Chen, X. (2006). Multi-relaxation-time lattice Boltzmann model for incompressible flow. Physics Letters A, 359, 564-572.

Fell, R., \& Fry, J.-J. (2007). The state of the art of assessing the likelihood of internal erosion of embankment dams, water retaining structures and their foundations. In R. Fell \& J.-J. Fry (Eds.), Internal Erosion of Dams and Their Foundations (pp. 1-23). Leiden: EDF, IREX, Taylor and Francis/Balkema.

Fell, R., Foster, M. A., \& Wan, C. F. (2007). A framework for assessing the likelihood of internal erosion and piping of embankment dams and their foundations. In R. Fell \& J.-J. Fry (Eds.), Internal Erosion of Dams and Their Foundations (pp. 65-70). Leiden: EDF, IREX, Taylor and Francis/Balkema.

Frisch, U. (1989). Relation between the Lattice Boltzmann equation and the Navier-Stokes equations. Physica D, 47, 231-232.

Frisch, U., d'Humières, D., Hasslacher, B., Lallemand, P., Pomeau, Y., \& Rivet, J. P. (1987). Lattice gas hydrodynamics in two and three dimensions. Complex Systems, 1, 649-707.

Frisch, U., Hasslacher, B., \& Pomeau, Y. (1986). Lattice-gas automata for the Navier-Stokes equation. Physical Review Letters, 56, 1505-1508.

He, X., \& Luo, L.-S. (1997a). A priori derivation of the lattice Boltzmann equation. Physical Review E, 55, 6333-6336.

He, X., \& Luo, L.-S. (1997b). Lattice Boltzmann model for the incompressible Navier-Stokes equation. Journal of Statistiscal Physics, 88, 927-944.

Ladd, A. J. C. (1994a). Numerical simulations of particulate suspensions via a discretized Boltzmann equation. Part 1. Theoretical foundation. Journal of Fluid Mechanics, 271, 285-309.

Ladd, A. J. C. (1994b). Numerical simulations of particulate suspensions via a discretized Boltzmann equation. Part 2. Numerical results. Journal of Fluid Mechanics, 271, 311-339. 
Lallemand, P. \& Luo, L.-S. (2000). Theory of the lattice Boltzmann method: Dispersion, dissipation, isotropy, Galilean invariance, and stability. Physical Review E, 61, 6546-6562.

Lallemand, P., \& Luo, L.-S. (2003). Lattice Boltzmann for moving boundaries. Journal of Computational Physics, 184, 406-421.

Lominé, F., Scholtès, L., Sibille, L., \& Poullain, P. (2013). Modeling of fluid-solid interaction in granular media with coupled lattice Boltzmann/discrete element methods: Application to piping erosion. International Journal for Numerical and Analytical Methods in Geomechanics, 37, 577596.

Mansouri, M., Delenne, J., El Youssoufi, M., \& Seridi, A. (2009). Dem-lbm approach to the onset of sand boiling. AIP Conference Proceedings, 1145, 1009-1012.

McNamara, S., Flekkøy, E. G., \& Måløy, K. J. (2002). Grains and gas flow: Molecular dynamics with hydrodynamic interactions. Physical Review E, 61, 4504-4509.

McNamara, G. R., \& Zanetti, G. (1988). Use of the boltzmann equation to simulate lattice-gas automata. Physical Review Letters, 61, 2332-2335.

Nguyen, N.-Q., \& Ladd, A. J. C. (2003). Lubrication corrections for lattice-Boltzmann simulations of particle suspensions. Physical Review E, 66, 046708.

Papamichos, E., \& Vardoulakis, I. (2005). Sand erosion with a porosity diffusion law. Computers and Geotechnics, 32, 47-58.

Qian, Y.H., D’Humières, D., \& Lallemand, P. (1992). Lattice BGK models for Navier-Stokes equation. Europhysics Letters, 17, 479.

Radjai, F., \& Dubois, F. (2011). Discrete-element modeling of granular materials. Hoboken, NJ: Wiley-ISTE.

Rotunno, A., Callari, C., \& Froiio, F. (in preparation). A finite element method for localized erosion in porous media with applications to backward erosion piping in levees. Journal paper in preparation.

Roux, J.-N., \& Chevoir, F. (2005). Discrete numerical simulation and the mechanical behavior of granular materials. Bulletin des laboratoires des ponts et chaussées, 254, 109-138.

Schäfer, M., Turek, S., Durst, F., Krause, E., \& Rannacher, R. (1996). Benchmark computations of laminar flow around a cylinder. In E. Hirschel (Ed.), Notes on numerical fluid mechanics: Flow simulation with high-performance computers (pp. 547-566). Braunschweig: Vieweg.

Sellmeijer, H., de la Cruz, J. L., van Beek, V. M., \& Knoeff, H. (2011). Fine-tuning of the backward erosion piping model through small-scale, medium-scale and IJkdijk experiments. European Journal of Environmental and Civil Engineering, 15, 1139-1154.

Sibille, L., Lominé, F., Poullain, P., Sail, Y., \& Marot, D. (2015). Internal erosion in granular media: Direct numerical simulations and energy interpretation. Hydrological Processes, 29, 2149-2163.

Tomac, I., \& Guierrez, M. (2013). Discrete element modeling of non-linear submerged particle collisions. Granular Matter, 15, 759-769.

van Beek, V. M., Knoeff, H., \& Sellmeijer, H. (2011). Observations on the process of backward erosion piping in small-, medium-and full-scale experiments. European Journal of Environmental and Civil Engineering, 15, 1115-1137.

Vardoulakis, I., Papanastasiou, P., \& Stavropoulou, M. (2001). Sand erosion in axial flow conditions. Transport in Porous Media, 45, 267-280.

Vardoulakis, I., Stavropoulou, M., \& Papanastasiou, P. (1996). Hydro-mechanical aspects of the sand production problem. Transport in Porous media, 22, 225-244.

Wachs, A. (2009). A DEM-DLM/FD method for direct numerical simulation of particulate ows: Sedimentation of polygonal isometric particles in a Newtonian fluid with collisions. Computures \& Fluids, 38, 1608-1628.

Wan, C. F., \& Fell, R. (2002). Investigation of internal erosion and piping of soils in embankment dams by the soil slot erosion test and the hole erosion test. Sydney: School of Civil and Environmental Engineering, University of New South Wales.

Wan, C. F., \& Fell, R. (2004). Laboratory tests on the rate of piping erosion of soils in embankment dams. Geotechnical Testing Journal, 27, 295-303. 
Wang, D., Fu, X., Jie, Y., Dong, W., \& Hu, D. (2014). Simulation of pipe progression in a levee foundation with coupled seepage and pipe flow domains. Soils and Foundations, 54, 974-984.

Wolfram, S. (1994). Cellular automata and complexity: Collected papers. Reading, MA: AddisonWesley.

Yang, F.-L., \& Hunt, M. (2008). A mixed contact model for an immersed collision between two solid surfaces. Philosophical Transactions of the Royal Society A, 366, 2205-2218.

Yen, B. C., \& Tung, Y.-K. (1993). Reliability and uncertainty analyses in hydraulic design. New York, NY: ASCE Publications.

Yu, D., Mei, R., Luo, L.-S., \& Shyy, W. (2003). Viscous flow computations with the method of lattice Boltzmann equation. Progress in Aerospace Sciences, 39, 329-367.

Zeghal, M., \& El Shamy, U. (2002). A continuum-discrete hydromechanical analysis of granular deposit liquefaction. International Journal for Numerical and Analytical Methods in Geomechanics, 28, 1361-1383.

Zhang, X., Wong, H., Leo, C., \& Bui, T. (2013). A thermodynamics-based model on the internal erosion of earth structures. Geotechnical and Geological Engineering, 31, 479-492.

Zhang, L., Xu, Y., \& Jia, J. (2009). Analysis of earth dam failures: A database approach. Georisk, 3, 184-189.

Zou, Q., \& He, X. (1997). On pressure and velocity boundary conditions for the lattice Boltzmann BGK model. Physics of Fluids, 9, 1591-1598. 